\title{
Accounting for small-scale heterogeneity and variability of clay rock in homogenised numerical micromechanical response and microcracking
}

\author{
Benoît Pardoen · Pierre Bésuelle · Stefano Dal Pont · Philippe Cosenza · Jacques \\ Desrues
}

Received: date / Accepted: date

\begin{abstract}
Clayey rocks have a complex microstructure with multiple characteristic lengths. Deformation under mechanical loading generally induces damage by microcracking, which essentially concerns the scale of mineral inclusions embedded in the clay matrix. The modelling of these materials is considered within the framework of a double scale approach, by numerical homogenisation, of the squared finite element method type. This allows a heterogeneous microstructure of the material to be taken into account and a distribution of morphological properties to be introduced. Emphasis is placed on the generation of microstructures satisfying experimental observations, and keeping a certain simplicity to fit into the framework of double scale modelling. The material characteristics and behaviour are defined at the grain scale: the mineralogical properties include the mineral phase proportions and the grain morphology, while the material constituents are represented by elastic grains separated by damageable cohesive crack models. Then, the overall microscale behaviour of the material under solicitation is derived from equilibrated elementary area (EA) configuration and computational homogenisation. The variability of the material response is studied with regard to small-scale aspects as microstructure variability, microstructure size, grain angularity, and properties of grain contacts. Deformation anal-
\end{abstract}

\section{B. Pardoen}

Univ. de Lyon, LTDS, ENTPE, 69120 Vaulx-en-Velin, France ; formerly (at time of research) at Univ. Grenoble Alpes, CNRS, Grenoble INP, 3SR, 38000 Grenoble, France

E-mail: benoit.pardoen@entpe.fr

B. Pardoen · P. Bésuelle · S. Dal Pont · J. Desrues

Univ. Grenoble Alpes, CNRS, Grenoble INP, 3SR, 38000 Grenoble, France

P. Cosenza

Univ. de Poitiers, IC2MP, 86000 Poitiers, France yses at grain contacts emphasise a dominant shear deformation mode and the development of decohesion between grains. The latter induces microfaulting processes across the entire EA and strain softening of the overall response. Moreover, the improvement of microscale behaviour modelling opens new possibilities for more realistic multi-scale modelling and upscaled behaviour of heterogeneous rocks.

Keywords Micromechanics · Numerical modelling · Clay rock $\cdot$ Microcracking $\cdot$ Homogenised response

\section{Introduction}

Clayey rocks are a class of rocks considered for different geological sites for the deep storage of nuclear wastes, due to their low permeability property and resealing capacity (Andra 2005; Croisé et al. 2004; Kickmaier and McKinley 1997). Understanding the role of microstructure on these properties, and more generally on the bulk mechanical behaviour and multiphysical couplings at the macroscopic scale (that of the engineer) is an important issue. The investigation of the small-scale material behaviour is a major objective to explain mechanisms observed at larger scale (e.g., Morgenstern and Tchalenko 1967; Rutter et al. 1986; French et al. 2015; Haines et al. 2013).

Clayey rocks have the particularity of having a complex microstructure, which extends over a wide range of characteristic lengths, from the nanometric scale of clay crystals to the millimetric scale of mineral inclusions, or even the centimetric to metric scale of variations in mineralogical compositions (Ortega et al. 2007; Yven et al. 2007; Klinkenberg et al. 2009; Robinet et al. 2012). Depending on the physical phenomena studied, the relevant scales are not the same. Concerning the mechanical damage, the permeability variation due to deformation and even the macroscopic failure (due to a spatial localisation of the damage), the scale of 
mineral inclusions, which is that of microcracking, is the most important (Desbois et al. 2017; M'Jahad et al. 2017). At this micrometric scale, mineral inclusions of different kinds appear to be embedded in a clay matrix. The material is disordered with regard to the distribution, nature and morphology of the mineral inclusions. A statistical description of the microstructure makes it possible to extract average characteristics (Fauchille 2015; Cosenza et al. 2015a,b, 2019; Fauchille et al. 2018). However, the microstructure of these materials is not perfectly periodic and the spatial distribution of mineral inclusions cannot be completely determined, it appears that it conserves a part of spatial variability.

The transition of scale between the microstructure of the material and the macroscopic scale, between the statistically representative elementary volume (REV) and the equivalent homogeneous medium, has seen the development of many approaches since several decades (e.g., Zaoui 2002, for a survey). In recent years, due to advances in digital technology, new multiscale approaches have emerged (e.g., Geers and Yvonnet 2016), in particular computational homogenization methods (Smit et al. 1998; Feyel and Chaboche 2000; Kouznetsova et al. 2001; Galvanetto and Aliabadi 2009; Schröder 2014). They improve the microstructure definition with more realistic morphologies, interface consideration and damage. The method consists of a numerical treatment of both micro and macro scale boundary value problems (BVP), with a dialog between the two computations. Some macro variables (e.g., strain) are transferred to the micro-BVP to determine the boundary conditions. In return, the macro dual quantities (e.g., stress) and tangent stiffness matrix result from an averaging of the solution of the micro-BVP. Finite element method (FEM) is generally used at macro-scale and the micro scale problem can be solved also by FEM (referred as $\mathrm{FE}^{2}$ method) or by other methods, for example discrete element method (Desrues et al. 2019). The method used in this work is a $\mathrm{FE}^{2}$ approach where hydro-mechanical coupling can be introduced at both scales (Frey et al. 2013; Pardoen et al. 2018; van den Eijnden et al. 2016).

In these two-scale approaches, the model response depends entirely on the microstructure: first, the spatial description of the heterogeneity of multiphase arrangement, then, the constitutive behaviour of phases and interfaces. The digital microstructure can be built on realistic geometric models obtained directly from images by Scanning Electron Microscopy (SEM) or X-ray Computed Tomography (CT) (e.g., Sonon et al. 2015; Nguyen et al. 2017; Stamati et al. 2019). These methods require fine mesh sizes and result in calculation costs for the micro scale alone that are not compatible with a double-scale calculation. This means that the microstructure must be generated in an artificial way, to remain both simple (easy to mesh with few elements) and respect a certain realism. Generation methods such as random sequential addition (Widom 1966; Cooper 1988), discrete element method (Lubachevsky and Stillinger 1990; Ghossein and Lévesque 2013), or Voronoi tessellation (Fritzen et al. 2009; Massart and Selvadurai 2012) can be used. This last one has been selected here because it is more adapted to the microstructure of clayey rocks.

Concerning the modelling of the micromechanical behaviour of clayey rocks, some recent studies have been developed with various homogenization methods. They include for instance damage, inclusion debonding, inherent anisotropy, and plastic compressible porous clay matrix (e.g., Shen et al. 2012; Shen and Shao 2014, 2015). Based on deformation and cracking observed at microscale, similar mechanisms will be embedded in the definition of the digital microstructure.

The article first describes the material studied, which is a Callovo-Oxfordian clay rock, and its microstructure. Then we briefly present the $\mathrm{FE}^{2}$ model used, derived from the work of Frey et al. (2013) and van den Eijnden et al. (2016). The microstructure generation method, based on the Voronoi method, has been adapted for our case where mineral inclusions, according to the mineralogical phases, do not have the same properties in terms of granulometry, elongation, angularity, and preferential orientation. Then, we present microstructures generated to satisfy the experimental statistical data. We explore the question of the representativity of elementary areas and the variability with respect to the mechanical response of models.

\section{Microstructure of the Callovo-Oxfordian clay rock}

The Callovo-Oxfordian clay rock (COx) is the host rock studied at the ANDRA Underground Research Laboratory (URL) located in Bure (Meuse-Haute Marne, eastern France) at approximately 490m below ground surface (Andra 2005; Armand et al. 2014, 2017). It is a sedimentary, low-permeability, cross-anisotropic, and indurated shale exhibiting quasi-horizontal bedding planes. The microstructure of the COx clay rock is heterogeneous and composed of several types of minerals.

Experimental observations and characterisations of the rock microstructure (Yven et al. 2007; Robinet et al. 2012; Cosenza et al. 2015a,b, 2019; Desbois et al. 2017) are considered to define the material in a realistic manner. Hereafter, the focus is mainly on the description of mineralogical constituents, grain morphology (i.e., geometrical characteristics), and microstructural patterns. The analysis methods on micro- to meso-structures include SEM, X-ray micro-CT, and image analysis with segmentation techniques. Measurements, as those in Fig. 1, lead to the determination of mineral groups, spatial distributions, mineral area fractions, as well as size, shape, and orientation of mineral inclusions. 


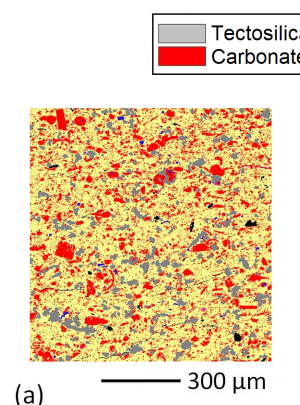

(a)

(b)

Heavy minerals Clay matrix

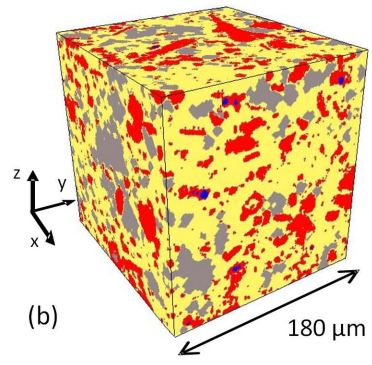

Fig. 1 Microstructure of COx clay rock with spatial distribution of mineral groups: (a) 2D SEM segmented image perpendicular to the bedding planes and (b) 3D micro-CT subvolume (EST26095) (Robinet et al. 2012).

\subsection{Mineralogical composition}

Experimental data indicate that the Callovo-Oxfordian clay rock is composed of (Fig. 1): tectosilicates $\left(-\mathrm{Si}_{x} \mathrm{O}_{y}\right.$, mainly quartz, $10-40 \%)$, carbonates $\left(-\mathrm{CO}_{3}\right.$, mainly calcite, $\left.15-80 \%\right)$, heavy minerals $\left(\mathrm{FeS}_{2}\right.$, pyrite, in a low proportion of $\left.0-3 \%\right)$, and clay minerals (20-60\%) as described by Gaucher et al. (2004); Andra (2005); Robinet et al. (2012); Armand et al. (2017). The clay minerals consist mainly of illite and interstratified illite/smectite. In situ, the proportions of the different minerals in the rock layer show some variability within the stratigraphic levels (i.e. with the depth) (Armand et al. 2014, 2017). The COx geological layer is composed of units rich in clay minerals (main part of the total layer thickness) and rich in carbonates.

In the following, the zone of interest is the median depth of the COx layer $(-490 \mathrm{~m})$ corresponding to the main level of the ANDRA URL. It is located in the clay unit which exhibits a high clay mineral content with non-porous mineral inclusions (mostly calcite and quartz) embedded in a porous clay matrix (Robinet et al. 2012). Its mineralogical composition is: clay fraction 40-60\% (Armand et al. 2017), calcite $25-35 \%$, quartz $15-25 \%$, and minor feldspar, mica and pyrite (Gaucher et al. 2004). The scattering of the local clay fraction based on experimental data from Cosenza et al. (2015a,b) is illustrated in Fig. 6 (b) where it is compared to numerical results (see sections 4.1 and 4.2). The local clay fraction and its scattering are measured on square cells having a dimension (critical length) of $110 \mu \mathrm{m}$.

At this scale (i.e. inclusion scale), the only visible porosity is the macroporosity (macropores), the rest of the porosity being in the clay matrix. However, macropores are very much in the minority with a content less than a few percent. This was experimentally measured by Robinet et al. (2012) based on SEM images with macropores content $<2 \%$.

\subsection{Size and morphology of mineral inclusions}

The characteristics of mineral inclusions are often quantified by considering a representative population of grains. The inclusions have a size ranging from several micrometers to a few hundreds of micrometers (Sammartino et al. 2003; Robinet et al. 2012). The size is defined as two-dimensional intercepted area in material sections. In average, quartz grains are larger than carbonates, and pyrite grains are the smallest. The complete granulometry of quartz and carbonate inclusions can be found in Robinet (2008). It allows to derive the mean area of inclusions which are about $100 \mu \mathrm{m}^{2}$ for quartz and $45 \mu \mathrm{m}^{2}$ for carbonates in the COx clay rock. It is to mention that the experimental distribution of inclusion area is a power law (Robinet 2008). Therefore, defining mean areas of inclusions is not straightforward and is realised in relation to the overall area of investigation.

Other geometrical characteristics of the mineral inclusions are defined by analyses of the small-scale structure. The anisotropy of granular materials due to petrogenesis processes is often quantified by the morphology of the grains. In material sections, their elongation and orientation are defined with an inclined ellipse fitting procedure on the grain intercepted areas (Yamaji and Masuda 2005; Robinet et al. 2012). The grain elongation index corresponds to the ratio of the ellipse minor $h$ and major $L$ axes:

$e=\frac{h}{L}$

and the grain orientation $\alpha$ corresponds to the angle between the major axis and the reference horizontal direction (i.e. the bedding plane direction).

Results of experimental measurements in sections perpendicular to the bedding planes are summarised hereafter (Robinet et al. 2012). The average values of grain elongation are of 0.64 and 0.55 for quartz and carbonates, respectively. This indicates that carbonate inclusions are more elongated than quartz. The average orientation of mineral inclusions is globally horizontal, even if the orientation of quartz is more variable than for carbonates. The frequency distributions of grain elongation and orientation are detailed further in section 4.2.3 (Robinet 2008; Robinet et al. 2012). The experimental characterisations have highlighted that the crossanisotropy of the material structure is due to the morphology and orientation of the mineral inclusions. They have an elongated shape with a preferential orientation parallel to the bedding planes. Moreover, mineralogical and microstructural average characteristics of COx clay rock are summarised in Table 1 based on the detailed experimental data. 
Table 1 Mineralogical and microstructural average characteristics of COx clay rock in vertical sections perpendicular to the bedding planes (EST26095).

\begin{tabular}{lllll}
\hline Minerals & Area fraction & Area & Elongation & Orientation \\
& {$[\%]$} & {$\left[\mu \mathrm{m}^{2}\right]$} & $e[-]$ & $\left.\alpha{ }^{\circ}\right]$ \\
\hline Tectosilicates (quartz) & 18 & 100 & 0.64 & 0 (variable) \\
Carbonates (calcite) & 30 & 45 & 0.55 & 0 \\
Heavy minerals (pyrite) & 2 & - & - & - \\
Clay matrix & 50 & - & - & - \\
\hline
\end{tabular}

\subsection{Deformation and cracking mechanisms at microscale}

Even if deformation mechanisms are dominantly cataclastic, observations from experimental investigations at microscale with microscopy techniques (e.g. SEM) indicated that crystal plastic mechanisms occur (Desbois et al. 2017). Potential decohesion mechanisms around inclusions and cracking within the clay matrix develop in the clay rock (Desbois et al. 2017; Wang et al. 2015; Bésuelle et al. 2019). The interfaces between (non-clay) mineral inclusions and clay matrix play the role of microcracking attractors. The microcracking paths develop (by microcracks coalescence) between the inclusions and goes, from grains to grains, through the clay matrix (intergranular cracks) (Desbois et al. 2017). Breakage of inclusions can also occur in quartz and calcite grains (intragranular cracks).

Furthermore, clay rocks exhibit heterogeneities at a lower scale (i.e. clay particles and platelets). Microcracks within the clay matrix at a lower scale would take their origin from these smaller scale heterogeneities.

\section{Microscale model}

The micro-scale model is part of the double scale $\mathrm{FE}^{2}$ approach, it serves as the constitutive model of the macro-scale simulation (Frey et al. 2013; Pardoen et al. 2018; van den Eijnden et al. 2016). The bridge between the two scales is realised by the periodic boundary conditions of the micro model that are a direct result of the macro-strain. The solution of the micro BVP allows to compute a homogenised stress tensor, as the response of the behaviour at the macroscale. We focus here on the micro-scale model that is devoted to the reproduction of the clayey rock behaviour. The material representation includes the heterogeneity of the microstructure at grain scale in the definition of a two-dimensional representative elementary area (EA). Therefore, the microscale behaviour is modelled as interacting deformable grains corresponding to the different mineral phases.

A more realistic representation of the material microstructure would be a three-dimensional model. However, before going to more realistic 3D representation of the material, 2D calculations constitute reference results on which analyses of 3D calculations can be based on later. Moreover, the $2 \mathrm{D}$ microstructure is enriched hereafter (section 4 ) by tak- (a)

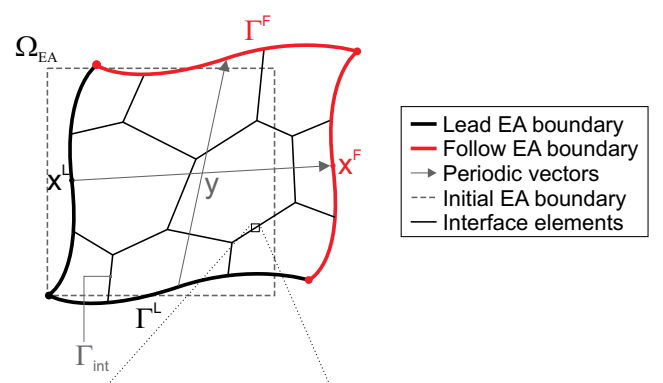

(b)

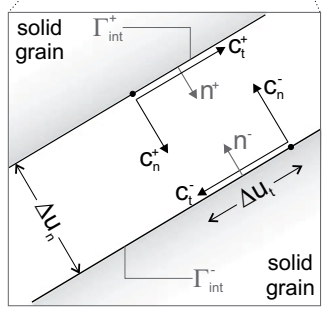

Fig. 2 Microscopic mechanical model: (a) deformed periodic microstructure and (b) boundary cohesive forces at grain interface (Marinelli et al. 2016).

ing into account realistic properties of the mineral inclusions (morphology, area fraction, orientation), measured experimentally in $2 \mathrm{D}$ material sections.

\subsection{Elementary area definition}

The microscale elementary area contains the details of the material microstructure. Its role is to reproduce the behaviour and deformation processes at small scale. The model is twodimensional under plane strain conditions (van den Eijnden et al. 2017). The EA is composed of an assembly of elastic deformable continuous solid grains, representing the components of the material, with damageable cohesive interfaces (Fig. 2 (a)). The hydro-mechanical coupling is neglected in this paper. The non linear behaviour is concentrated at grain contacts, with possible strain softening. As aforementioned, macropores are very much in the minority at this scale (Robinet et al. 2012); therefore, their effect is neglected and only the crack porosity is considered. The microscale BVP is solved by finite element method, under small transformation assumption. The EA length scale is assumed to be much smaller than the macroscale problem, to satisfy the separation of scales.

\subsection{Boundary conditions}

To be representative of material behaviour at larger scale, the EA assumes a periodic microstructure with periodic boundary conditions (Frey et al. 2013; Marinelli et al. 2016; van den Eijnden et al. 2016, 2017). 
The macroscale deformation gradient tensor $\mathbf{F}^{\mathrm{M}}=1+$ $\nabla u^{\mathrm{M}}$ is decomposed in a rotational component $\mathbf{R}^{\mathrm{M}}$ and a symmetric stretch component $\mathbf{U}^{\mathrm{M}}$ :

$F_{i j}^{\mathrm{M}}=R_{i k}^{\mathrm{M}} U_{k j}^{\mathrm{M}}$

The macroscale small strain tensor $\varepsilon^{\mathrm{M}}=\mathbf{U}^{\mathrm{M}}-\mathbf{I}$ is used to determine the periodic boundary condition of the microscale BVP, while the homogeneised stress tensor is rotated back using the rotation tensor $\mathbf{R}^{\mathrm{M}}$.

This implies linked kinematics of homologous points on the opposite sides of external boundary of the elementary area (Fig. 2 (a)). Introducing lead point $x_{i}^{L}$ and follow point $x_{i}^{F}$, the periodic constrain between homologous points on the microscale displacement $u^{m}$ is:

$u_{i}^{m, F}=u_{i}^{m, L}+\varepsilon_{i j}^{\mathrm{M}} y_{j}$

where $y_{i}=x_{i}^{F}-x_{i}^{L}$ is the periodic vector. The associated antiperiodic condition on the boundary traction forces $t_{i}$ are:

$t_{i}^{F}+t_{i}^{L}=0$

Concerning the internal grain boundaries, they are subdivided into lower and upper parts $\Gamma_{i n t}^{-/+}$of outward normal $n^{-/+}$(Fig. 2 (b)). Reciprocal cohesive forces $c_{i}^{-/+}$act on the two parts which displacements are $u_{i}^{-/+}$, leading to displacement discontinuity $\Delta u_{i}=u_{i}^{+}-u_{i}^{-}$across the interface. The interface behaviours in normal and tangential directions are detailed in section 3.5.

\subsection{Balance equations}

The field equation for the conservation of momentum of the microscale BVP is written in the weak form for any kinematically admissible virtual field of displacement $u^{\star}$ as follows:

$\int_{\Omega_{E A}} \sigma_{i j}^{m} \frac{\partial u_{i}^{\star}}{\partial x_{j}} d \Omega=\int_{\Gamma_{i n t}^{+}} c_{i}^{+} u_{i}^{\star,+} d \Gamma+\int_{\Gamma_{i n t}^{-}} c_{i}^{-} u_{i}^{\star,-} d \Gamma$

Note that gravity effect is not considered at microscale.

The non-linear microscale equation system is solved by: enforcement of loading through the boundary conditions, linearisation of the field equations (Newton-Raphson iterative procedure), and FEM spatial discretisation. The discretisation of the solid grains is realised by two-dimensional four-node isoparametric quadrilateral FEs with four integration points. The one-dimensional interface elements are composed of four nodes with initially zero thickness (van den Eijnden et al. 2016). The solution allows to determine the unknown field $u_{i}^{m}$ under equilibrium conditions.

\subsection{Computational homogenisation}

Computational homogenisation allows scale transition (upscaling) by deriving the homogenised response of the EAs. The homogenised variables are computed from the equilibrated EA configuration. It is based on Hill-Mandel macrohomogeneity condition (Hill 1965; Mandel 1972) of equal virtual work at both scales. Note that, for double scale $\mathrm{FE}^{2}$ computation, the consistent tangent operators are upscaled by static condensation (Kouznetsova et al. 2001), and rotated back using the rotation tensor $\mathbf{R}^{\mathrm{M}}$ (van den Eijnden et al. 2016).

For mechanical problems, the homogenised total stress tensor is obtained as follows:

$\sigma_{i j}^{\mathrm{M}}=\frac{1}{\Omega_{E A}} \int_{\partial \Omega_{E A}^{F}} t_{i} y_{j} d \Gamma$

It corresponds to an average of the microscopic stresses over the EA.

\subsection{Constitutive mechanical models}

The mechanical behaviour is characterised by assuming isotropic linear elastic solid phases separated by cohesive crack models (Bilbie et al. 2008; Frey et al. 2013). The latter are damageable cohesive interfaces allowing softening of the material due to deformation by solid grain movements. The cohesive linear damage models in the normal and tangential directions to the grain boundary are illustrated in Fig. 3. The independent expressions consist of bilinear laws with a first elastic part followed by a softening (damage) branch (Frey et al. 2013; Marinelli et al. 2016; van den Eijnden et al. 2016):

$$
\begin{aligned}
c_{n}^{t} & =c_{n}^{\max }\left(1-D_{n}^{t}\right) \frac{1}{D_{n}^{t}} \frac{\Delta u_{n}^{t}}{\delta_{n}^{c}} & & \text { if } \Delta u_{n}^{t} \geq 0 \\
& =c_{n}^{\max }\left(1-D_{n}^{t}\right) \frac{1}{D_{n}^{t}} \frac{\Delta u_{n}^{t}}{\delta_{n}^{c}}-\kappa \Delta u_{n}^{t^{2}} & & \text { if } \Delta u_{n}^{t}<0
\end{aligned}
$$

$c_{t}^{t}=c_{t}^{\max }\left(1-D_{t}^{t}\right) \frac{1}{D_{t}^{t}} \frac{\Delta u_{t}^{t}}{\delta_{t}^{c}}$

where $n$ and $t$ subscripts indicate the normal and tangential directions, $c_{t / n}^{t}$ are the interface cohesion forces at time $t$, $c_{t / n}^{\max }$ are the maximal cohesions, $\Delta u_{t / n}^{t}$ are the non-dimensional interface relative displacements (relative to the unit EA size) at time $t$. Further, $0 \leq D_{t / n}^{t} \leq 1$ are softening parameters corresponding to the relative degradation of the interfaces at time $t, \delta_{t / n}^{c}$ are the critical relative displacements for complete decohesion $\left(c_{t / n}=0, D_{t / n}=1\right)$. If interface closure occurs (grain normal contact), an unilateral boundary condition is considered with $\kappa>>c_{n}^{\max }$ as a penalisation term to avoid grain inter-penetration $\left(\Delta u_{n}<0\right)$. 

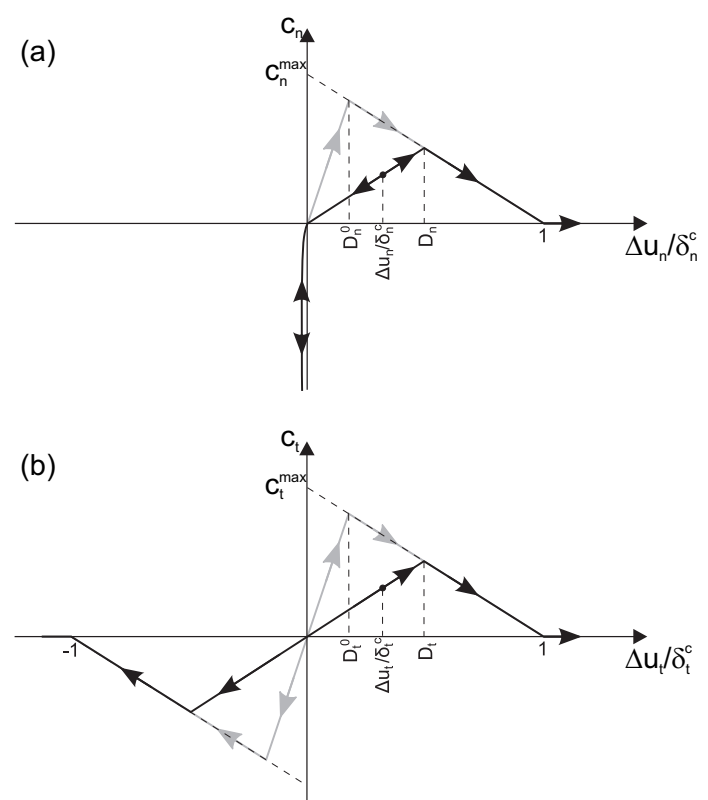

Fig. 3 Cohesive interface behaviour represented by linear damage laws in (a) normal and (b) tangential directions (van den Eijnden et al. 2016).

The interface state parameters $D_{t / n}^{t}$ depend on the time history $(\tau=0 \ldots \mathrm{t})$ of interface relative displacements $\Delta u_{t / n}$ and initial degradation states $D_{t / n}^{0}$ :

$$
\begin{aligned}
D_{n}^{t} & =\max \left(D_{n}^{0}, \frac{1}{\delta_{n}^{c}} \max _{0 \leq \tau \leq t}\left(\left|\Delta u_{n}^{\tau}\right|\right)\right) & & \text { if } \Delta u_{n}^{\tau} \leq \delta_{n}^{c} \\
& =1 & & \text { if } \delta_{n}^{c}<\Delta u_{n}^{\tau}
\end{aligned}
$$

(9)

$$
\begin{aligned}
D_{t}^{t} & =\max \left(D_{t}^{0}, \frac{1}{\delta_{t}^{c}} \max _{0 \leq \tau \leq t}\left(\left|\Delta u_{t}^{\tau}\right|\right)\right) & & \text { if }\left|\Delta u_{t}^{\tau}\right| \leq \delta_{t}^{c} \\
& =1 & & \text { if } \delta_{t}^{c}<\left|\Delta u_{n}^{\tau}\right|
\end{aligned}
$$

The interface elastic stiffnesses are affected by $D_{t / n}^{t}$ as follows:

$E_{t / n}^{t}=\frac{c_{t / n}^{t}}{\Delta u_{t / n}^{t}}=\frac{c_{t / n}^{\max }\left(1-D_{t / n}^{t}\right)}{D_{t / n}^{t} \delta_{t / n}^{c}}$

which is valid for $0 \leq \Delta u_{n}^{t} \leq D_{n}^{t} \delta_{n}^{c}$ (interface opening) and for $\left|\Delta u_{t}^{t}\right| \leq D_{t}^{t} \delta_{t}^{c}$. Following Eq. 11, $D_{t / n}^{0}$ define the initial interface stiffnesses $E_{t / n}^{0}$. The constant softening slopes of interface cohesion are given by:

$E_{t / n}=-\frac{c_{t / n}^{\max }}{\delta_{t / n}^{c}}$

With the above definitions of the micromechanical behaviour, the main part of the microstructure deformation is concentrated at grain contacts. Large deformations can therefore develop at the small scale and interfaces represent potential microcracks.

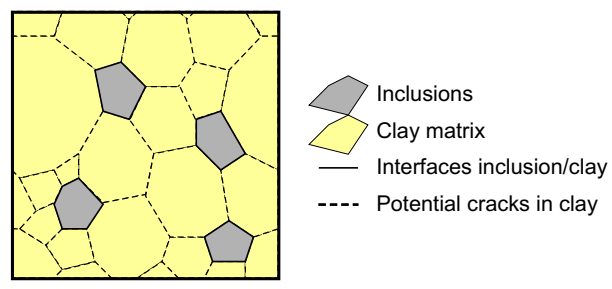

Fig. 4 Schematic representation of microstructural rupture modes by decohesion and crack mechanisms around the inclusions and within the clay matrix.

\section{Microstructure generation}

The microstructure of the COx clay rock (in clay-rich zone) is composed of non-porous mineral inclusions embedded in a porous clay matrix (Robinet et al. 2012). Representative EAs have to describe the grains but also the failure and damage modes at small scale. To do so, the interfaces are considered as potential microcracks and correspond to two types of failure, according to the mineralogy of the grains in contact: microcracks between two different mineral phases (inclusion-clay) and within the clay matrix (clay-clay). A schematic representation is illustrated in Fig. 4. Such scheme allows the developments of potential decohesion mechanisms around the inclusions and cracking within the clay matrix as observed in the clay rock (Desbois et al. 2017; Wang et al. 2015; Bésuelle et al. 2019). Because interface damage can develop in both tangential and normal directions, the rupture modes can be tensile/opening mode (mode I) or sliding shear mode (mode II). No inclusion breakage is considered in the representation, but can be easily introduced.

A multitude of potential microcrack paths is allowed within the clay matrix at the scale of the numerical elementary areas (EA), in adequacy with experimental observations. However, microcracking in clay at a lower scale would take its origin from smaller scale heterogeneities. Unfortunately, such level of complexity is not compatible with double-scale approaches. It is therefore not included in the model.

\subsection{Modified Voronoi tessellation}

One objective of the proposed approach is to consider a more realistic material microstructure for numerical doublescale scheme, based on experimental characterisation. Therefore, several characteristics of the COx clay rock are considered in the numerical definition of 2D microscale elementary areas. These characteristics are: the mineral phases and their area fractions; the size, elongation, orientation, and roundness of the mineral inclusions; as well as the characteristic size of representative elementary volumes (Robinet et al. 2012; Cosenza et al. 2015a).

The microstructures are generated with periodic $2 \mathrm{D}$ random Voronoi tessellation (Ghosh 2011). Each cell corre- 
sponds to a solid grain, representing either a mineral inclusion, either a clay grain of the clay matrix (Fig. 4). The characteristics of the mineral inclusions are taken into account in Voronoi diagrams adapted from the work of van den Eijnden et al. (2017). The previous generation algorithm allowed to generate EAs with heterogeneous and anisotropic characteristics. Nevertheless, it suffered a certain lack of representativeness of the microstructural characteristics. Improvements are realised by including several parameters in the algorithm that allow to control the grain morphology for each phase. The modifications of the EA generation method are described hereunder and in Fig. 5.

- Pre-tessellation - Mineral types (Fig. 5 (b)): Each cell site, also called seed, is assigned a solid phase type. The number of inclusions of each mineral type depends on granulometry and area fractions.

- Pre-tessellation - Distance condition (Fig. 5 (b)): An elliptical distance condition, specific to each mineral phase, is imposed around each site to define an exclusion zone for other seeds and thus contributes to defining the shape of the grain (elongation, orientation, and size). The characteristics of the ellipses correspond to a random variation around the average properties measured experimentally. More precisely, the variations of geometrical parameters of the exclusion elliptical zones follow uniform random distributions between lower and upper bounds. The latter are chosen in acceptable ranges regarding experimental measurements of grain morphology.

- Post-tessellation - Vertex adaptations (Fig. 5 (c-d)): After completing the tessellation, an oriented adaptation of the vertex positions of the cells is realised. This consists in moving the vertices between two grains, resulting from tessellation, away from the center of the largest grain. It allows to better reproduce the grain shape. A convexity verification of the cell shape is also performed to ensure a good quality of the FE mesh (Fig. 5 (e)). The FE mesh is defined automatically from the tessellation.

An example of a large and realistic microstructure of COx clay rock with 500 cells is visible in Fig. 5 (a). As aforementioned, a variability of the microstructural characteristics is allowed. It permits to reproduce the variability of mineral and clay fractions, elongation, and orientation (see section 4.2). Moreover, even if multiple potential microcrack paths are allowed in the EA, the crack location will depend on the Voronoi cell arrangement. This is required by the need to simplify the modelling of the microstructure to be compatible with a double-scale scheme. Nevertheless, the randomness of the generation allows to consider a population of microcracks from various microstructures.

\subsection{Representativeness}

The elementary areas that are considered are representative of (vertical) planes normal to the (horizontal) bedding planes. The representativeness of the microstructure is evaluated by comparison of numerical and experimental geometrical characteristics of the minerals. An analysis is carried out on a set of 100 EAs to assess representativeness and variability of the generation. The results demonstrate that a realistic definition of the material microstructure is considered by taking into account the geometrical characteristics of the mineral inclusions.

\subsubsection{Characteristic size}

The representativeness of the microstructure depends firstly on its size. The size of representative elementary volume (REV) measured experimentally for the COx clay rock is of $V_{R E V} \approx 0.001 \mathrm{~mm}^{3}$ giving a representative length of $L_{R E V} \approx$ $100 \mu \mathrm{m}$ (Robinet et al. 2012). Numerically, the EAs are dimensionless. However, if we assign a size to an inclusion based on experimental characterisations; then, we assign an artificial size to the EAs depending on the number of grains represented. An artificial dimension can thus be estimated for each EA by linking the number of cells to the size of the mineral inclusions by:

$\Omega_{E A}=L_{E A}^{2}=\frac{n_{E A, i n c l}}{\mu_{E A, \text { incl }}} S_{\text {incl }}$

where $\Omega_{E A}$ is the EA surface, $L_{E A}$ is the EA dimension, $n_{E A, i n c l}$ is the number of mineral inclusions in the EA, $\mu_{E A, i n c l}$ is the area fraction of inclusions in the EA, and $S_{\text {incl }}$ is the mean area of inclusions measured experimentally. Measurements being mainly available on quartz and carbonates (Table 1), the values $n_{E A, i n c l}, \mu_{E A, i n c l}$, and $S_{\text {incl }}$ are related to these minerals with $n_{E A, \text { incl }}=n_{E A, q u a r t z}+n_{E A, \text { carbonates }}, \mu_{E A, \text { incl }}=$ $\mu_{E A, q u a r t z}+\mu_{E A, \text { carbonates }}$ (Eq. 14), and $S_{\text {incl }}=51 \mu \mathrm{m}^{2}$ (derived from Robinet (2008) and Robinet et al. (2012)).

The number of 250 cells is then chosen to obtain an artificial EA dimension in agreement with the characteristic size of REV determined experimentally on morphological considerations. An analysis performed on a set of 100 generated random EAs leads to a mean value of $L_{E A}=105 \mu \mathrm{m}$, with minimal and maximal values ranging between 100 and $110 \mu \mathrm{m}$.

The same set of EAs is used hereafter to assess the representativeness of the microstructure generation. Examples of EAs are detailed in Fig. 6 (a) for different clay contents.

\subsubsection{Mineral phase contents}

Numerically, the mineral contents correspond to the area fractions of each mineral phase within a generated microstructure. It is calculated in an EA by summing the area of all 


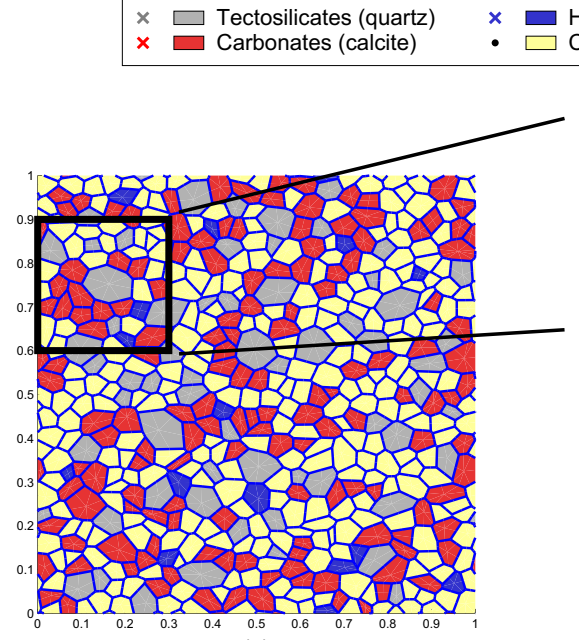

(a)

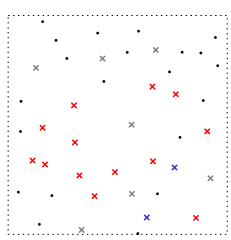

(b)

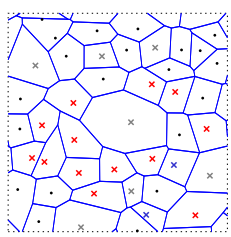

(d)
- Interfaces
- FE mesh

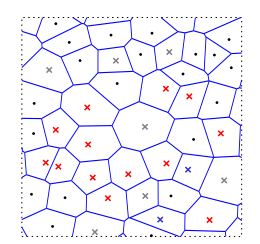

(c)

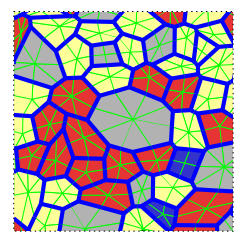

Fig. 5 Microstructure of COx clay rock represented by (a) a numerical periodic elementary area with the details of the generation steps: (b) Voronoi sites with distance condition, (c) tessellation, (d) vertex adaptations, (e) material assignment and FE meshing.

Voronoi cells belonging to each mineral type:

$\mu_{E A, \text { mineral }}=\frac{1}{S_{E A}} \sum_{i=0}^{n_{E A, \text { mineral }}} S_{\text {mineral }, i}$

where $n_{E A \text {,mineral }}$ is the grain number of a mineral type and $S_{E A}=1$ is the dimensionless size of the EA.

The frequency distributions of mineral area fractions are detailed in Fig. 6 (b). Only the results for pyrite is not presented because of the low number of inclusions. This is also the case for the other results presented further. The numerical average values are $18 \%$ of quartz, $30 \%$ of carbonates, $2 \%$ of pyrite, and $50 \%$ of clay matrix. They correspond to average values of experimental data (Table 1). Furthermore, numerical results and experimental measurements (Cosenza et al. 2015a,b) of the local clay fraction distribution are depicted in Fig. 6 (b). Their comparison can be realised because the sizes of the numerical EAs (mean value of $L_{E A}=$ $105 \mu \mathrm{m}$ ) and of the experimental measurement cells (critical length of $110 \mu \mathrm{m}$ ) are similar. It highlights that the scattering of the clay matrix content is also well captured in the set of numerical microstructures. This is achieved by improvements of the generation algorithm that allow to control the mineral area fractions.

\subsubsection{Morphology of mineral inclusions}

As aforementioned, the morphology of the mineral inclusions can be evaluated by ellipse fitting procedure. This procedure can also be applied to the cells of the numerical EAs. The frequency distributions of grain elongations and orientations of the quartz and carbonate inclusions are detailed in Fig. 7. The numerical results are compared to experimental data in sections perpendicular to the bedding planes (Robinet et al. 2012). The average numerical values of elongations

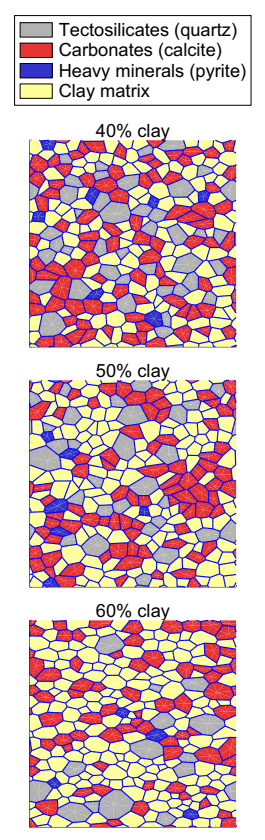

(a)

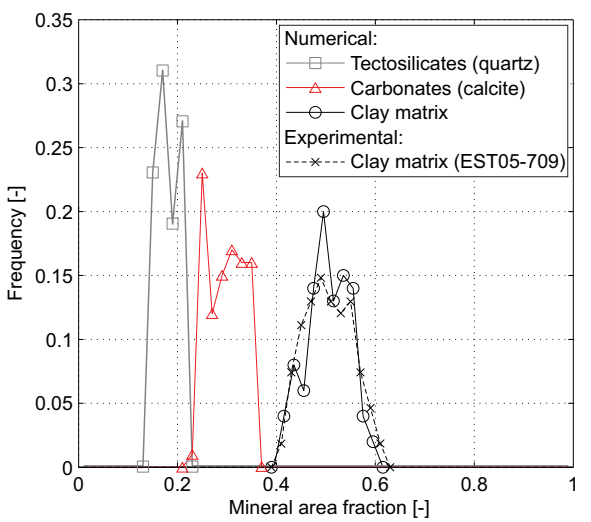

(b)
Fig. 6 Representative microstructures of COx clay rock: (a) examples of numerical elementary areas with various clay contents, and (b) distributions of mineral area fractions.

are 0.65 and 0.61 for quartz and carbonates, respectively. The preferential orientation of the inclusions in the EAs is horizontal and thereby parallel to the bedding planes. These results are in good agreement with average measurements (Table 1) and satisfactorily reproduces the variability of the characteristics.

In the examples of representative microstructures of Fig. 6 (a), one can observe that some grains are angular and others are more ellipsoidal. From microscale morphological observations, a non-negligible quantity of mineral grains in the 


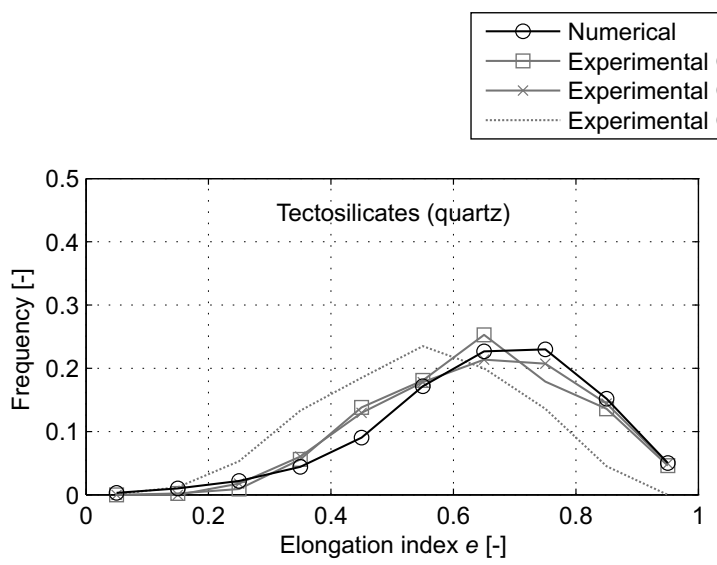

(a)

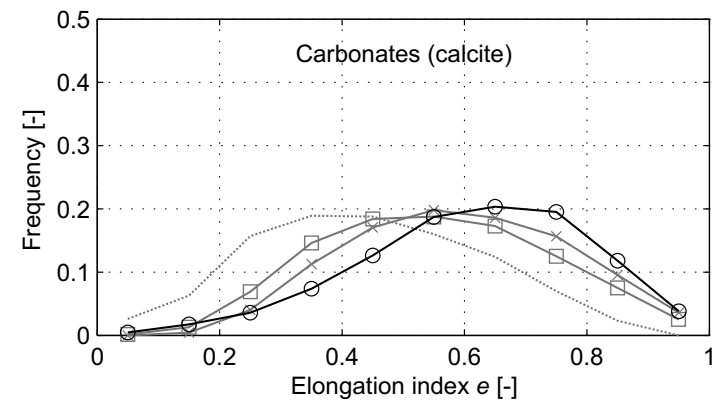

(c)

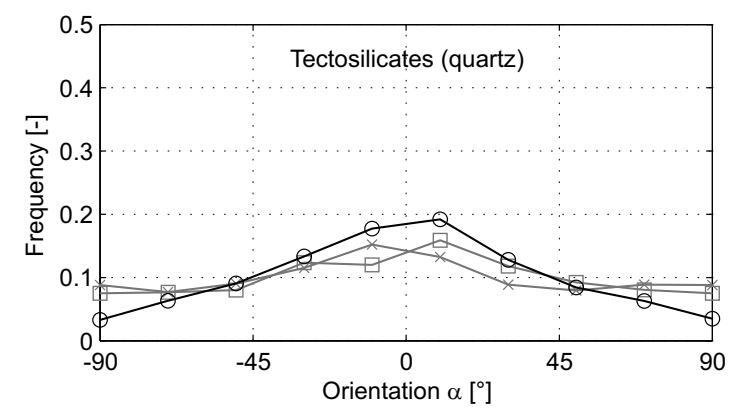

(b)

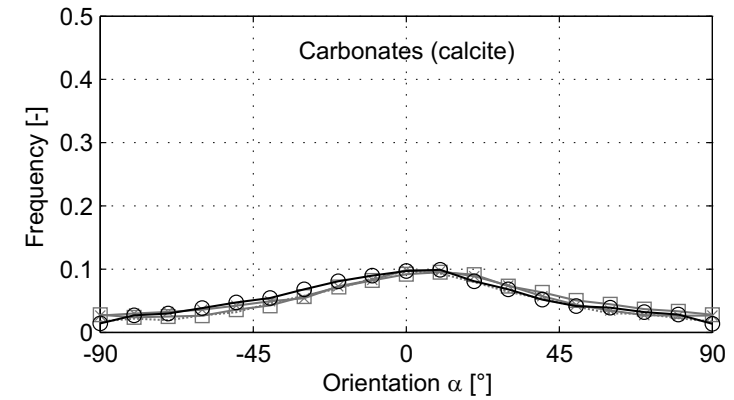

(d)

Fig. 7 Comparison of numerical and experimental morphological characteristics (Robinet 2008) of mineral inclusions in COx clay rock: (a,b) quartz, $(\mathrm{c}, \mathrm{d})$ carbonates, $(\mathrm{a}, \mathrm{c})$ elongation index, and $(\mathrm{b}, \mathrm{d})$ orientation.

clay rock can be assimilated to ellipsoids. Nevertheless, the ellipsoidal shape is far from being the most representative shape (Jorand 2006) and a certain number of concave grains are even observed. Consequently, there is no ideal geometric shape to represent the mineral grains and representative microstructures are generated to reproduce, as well as possible, the measurements of grain morphology.

\subsubsection{Size of mineral inclusions}

As for the EA size, an artificial size is assigned to the mineral grains by considering the mean area of quartz and carbonate inclusions measured experimentally, $S_{\text {incl }}$. The numerical grain size distributions of mineral inclusions of the entire set of EAs are detailed in Fig. 8. One can observe that the inclusion sizes range from several micrometers to a few hundreds. For each type of mineral, the minimal-meanmaximal sizes are: 6-72-230 $\mu^{2}$ for quartz, 6-43-115 $\mathrm{\mu m}^{2}$ for carbonates, and 6-35-84 $\mu \mathrm{m}^{2}$ for pyrite. Once again, these results are in good agreement with experimental data (Sammartino et al. (2003); Robinet et al. (2012); Table 1).

\section{Clay rock behaviour modelling}

Material behaviour is generally analysed through the response of samples subjected to enforced loading paths. The devel-

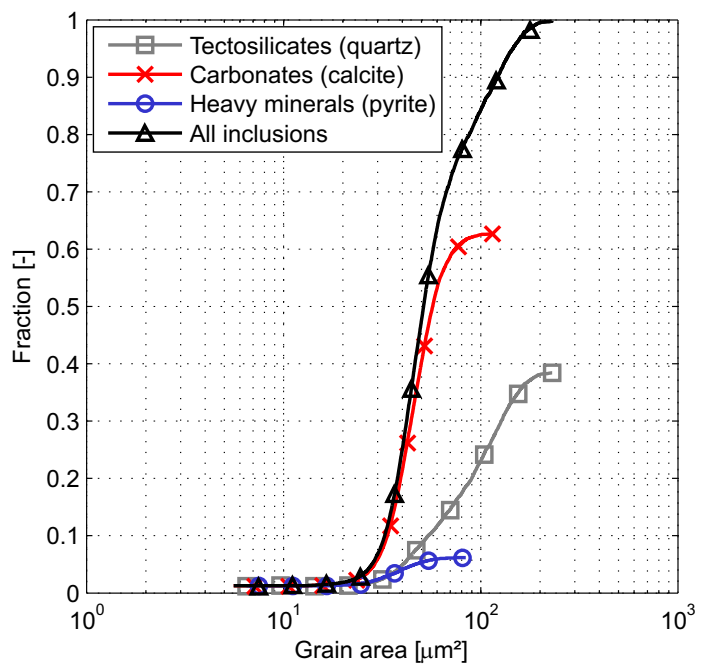

Fig. 8 Grain area distributions of numerical mineral inclusions in $\mathrm{COx}$ clay rock.

oped microscale model intends to address the COx clay rock behaviour at a the level of the clay matrix and of the mineral inclusions (with $L_{E A} \approx L_{R E V} \approx 100 \mu \mathrm{m}$ ). Therefore, numerical modelling of biaxial compression tests in plane strain state is performed on the 2D EAs to analyse the micromechanical behaviour of the clay rock. It consists of an isotropic confining phase up to a given $\sigma_{i n i}$ followed by a 
Table 2 Microscale mechanical parameters.

\begin{tabular}{lllll}
\hline Minerals & $E[\mathrm{GPa}]$ & $v[-]$ & & \\
\hline Tectosilicates (quartz) & 95 & 0.074 & & \\
Carbonates (calcite) & 84 & 0.317 & & \\
Heavy minerals (pyrite) & 305 & 0.154 & & \\
Clay matrix & 2.3 & 0.110 & & \\
\hline Interfaces & $\delta_{t / n}^{c}[-]$ & $D_{t / n}^{0}[-]$ & $c_{t}^{\max }[\mathrm{MPa}]$ & $c_{n}^{\max }[\mathrm{MPa}]$ \\
\hline & 0.1 & 0.001 & 2.5 & 1.0 \\
\hline
\end{tabular}

vertical compression with constant lateral stress. The latter is performed by enforcing a global homogenised strain in the vertical direction $\varepsilon_{11}$. The other components $\varepsilon_{13}$ and $\varepsilon_{33}$ are adjusted in order to maintain fixed the homogneised stress tensor components $\sigma_{33}=\sigma_{\text {ini }}$ and $\sigma_{13}=0$, since the answer is a priori anisotropic (iterative process by Newton-Raphson algorithm). An emphasis is put on the material response to deviatoric solicitation and on the deformation processes. Micromechanical properties are also calibrated against experimental data and the variability of the microscale behaviour is analysed.

\subsection{Micromechanical properties and response}

The EAs and their properties are chosen to reproduce the real microstructure of the clay rock, its micromechanical behaviour, and its deformation process. The mineral constituents are assigned linear elastic properties, with constitutive parameters depending on mineral phases of each grain. They are defined in Table 2 by Young's moduli $E$ and Poisson's ratios $v$ from Ahrens (1995); Robinet et al. (2012); van den Eijnden et al. (2017). The properties of the interfaces are assumed to be homogeneous, regardless of the mineralogical nature of the grains in contact. This is a simplifying hypothesis that will certainly have to be reviewed in the future.

Both mineral and interface properties influence the overall microscale response. The stiffnesses of grains and interfaces influence the initial stiffness of the response. The grains interface properties influence the microscale non-linear behaviour, peak strength, and strain softening (van den Eijnden et al. 2017). A calibration of interface properties is performed based on homogeneised microscale response of one EA to deviatoric loading. The chosen EA is the one on the bottom of Fig. 6 (a) with $60 \%$ clay content and the compression is performed with constant horizontal confining pressures of $\sigma_{33}=2$ and $12 \mathrm{MPa}$. The global stressstrain response curves and the calibration are detailed in Fig. 9. The curves are defined by the global deviatoric stress $q=\sigma_{11}-\sigma_{33}$ (given that $\sigma_{13}=0$ ) versus the homogenised vertical $\varepsilon_{11}$ and lateral $\varepsilon_{33}$ strains of the EA.

A comparison is made between the numerical results and experimental results of triaxial compression tests performed on laboratory specimen of COx clay rock (Armand et al. 2017; Pardoen and Collin 2017). It must be emphasised that the numerical modelling are performed at the microscale of an EA and the experimental tests were realised on a macroscopic scale (not to mention the $3 \mathrm{D}$ effects that are not taken into account in the modelling). The mechanical interactions between a set of EAs (which could constitute a macroscopic sample) are not taken into account in the numerical modelling. Thus, a correspondence between the behaviour at microscale (macro constitutive response) and experimental specimen (macro scale) is meaningful only for homogeneous modes of deformation. Non-homogeneous deformation modes at macroscale, induced for example by strain localisation, lead to a structural response which can no longer be compared directly to the constitutive behaviour (e.g., Bésuelle et al. 2007). It is well known that macroscale responses highlight a much more pronounced softening behaviour than the constitutive response, because of the non-homogeneous deformation. Comparing the deviatoric stress responses at micro and macro scales allows only the calibration of micromechanical properties up to the stress peak $q^{\max }$ (i.e. approximately the initialisation of the strain localisation).

Moreover, the macroscopic experimental results are given as references (for a further discussion and sensitivity analyses) in order to isolate the mechanical behaviour of only one EA and of the associated parameters (e.g. mineral contents, morphology of mineral grains, etc.)

The calibration is performed by analysing the influence of the interface parameters. The calibrated values are given in Table 2 and correspond to the curves marked with a number 0 in Fig. 9. The results indicate that the initial global stiffness, related to the mineral elastic properties and to the elastic stiffness of the interfaces, is well reproduced. The reproduction of the global non-linear behaviour and peak strength is analysed through the influence of the interface plastic parameters in both normal and tangential directions.

Fig. 9 (a) highlights the influence of the variation of maximal interface cohesion forces. From the values in Table 2 , the variation is performed by multiplying simultaneously $c_{t / n}^{\max }$ and $\delta_{t / n}^{c}$ by factors of $2,3 / 2,1,2 / 3,1 / 2$. The parameter sets are referenced in Table 3 . The results indicate that the peak strength of the overall microscale response is mostly related to the interface cohesion parameters. It is noted that the behaviour at $12 \mathrm{MPa}$ shows a higher resistance than at $2 \mathrm{MPa}$, typical of what is usually referred to as frictional macroscopic behaviour, while the behaviour of interfaces at the micro scale is only cohesive at this stage. This is because a high mean stress leads to greater difficulty in damaging the interfaces, at least in mode I, with contact forces reinforcing the effect of cohesion. Fig. 9 (b) highlights the influence of the variation of interface softening slopes. From the values in Table 2 , the variation is performed by simultaneously multiplying $\delta_{t / n}^{c}$ and dividing $D_{t / n}^{0}$ by factors of 4 , $2,1,1 / 2,1 / 4$ (Table 3 ). The results indicate that the post- 
(a)

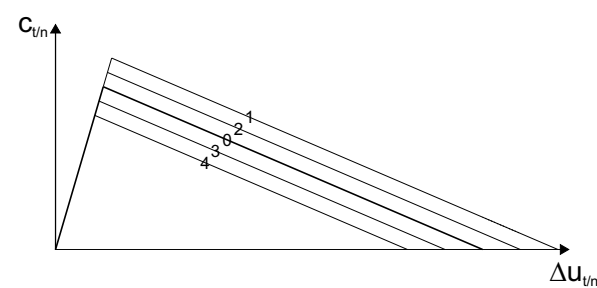

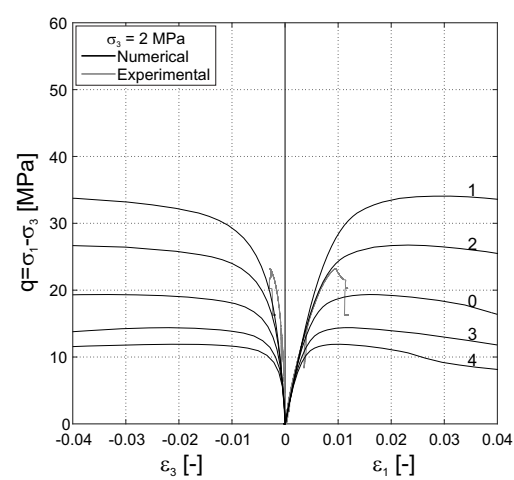
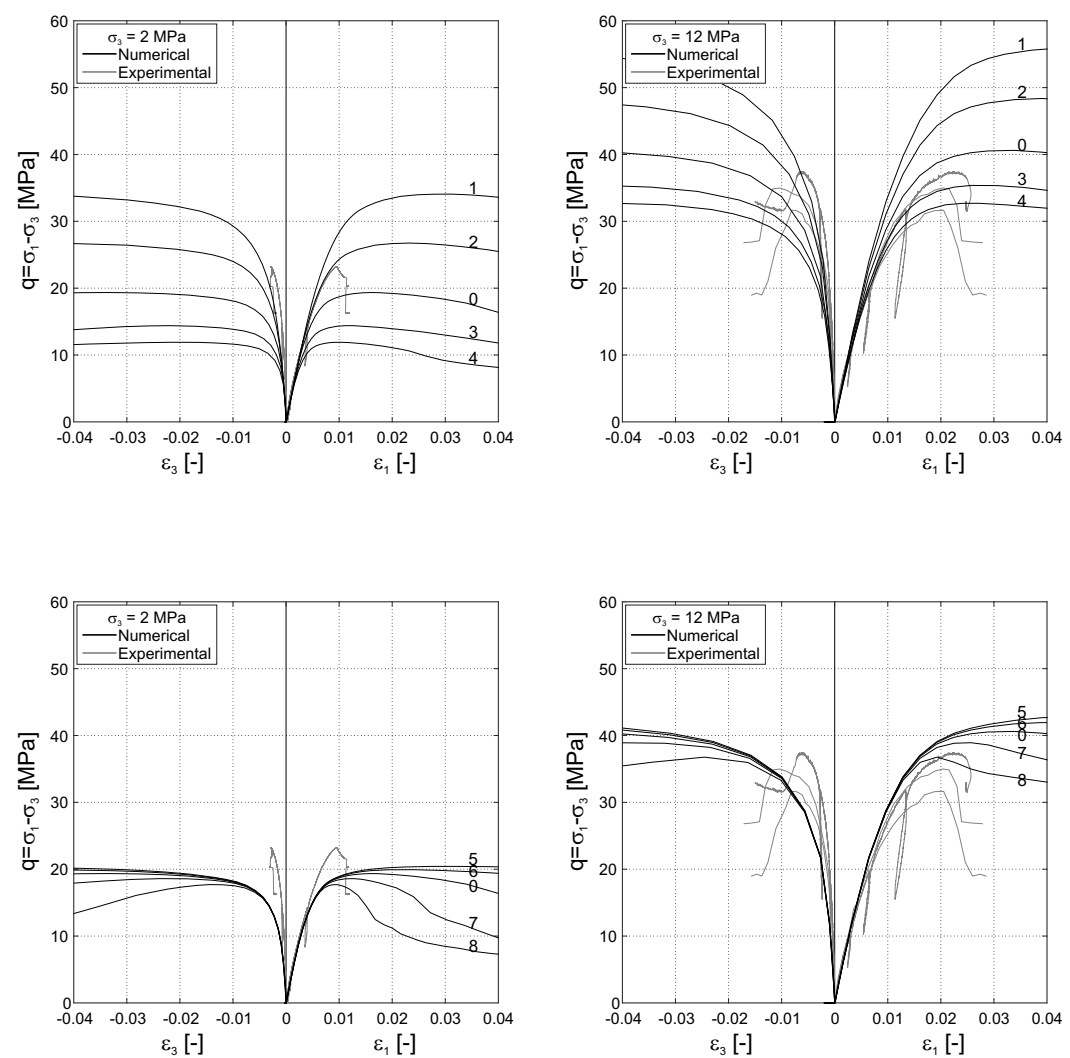

Fig. 9 Deviatoric stress responses at microscale under biaxial compression (numerical) compared to macroscale response under triaxial compression (experimental) for $\sigma_{3}=2$ and $12 \mathrm{MPa}$ : influence of (a) maximal interface cohesion forces and (b) interface softening slopes.

Table 3 Sets of grain interface parameters used for calibration with variation of maximal cohesion forces and softening.

\begin{tabular}{lllll}
\hline Parameter set & $\delta_{t / n}^{c}[-]$ & $D_{t / n}^{0}[-]$ & $c_{t}^{\max }[\mathrm{MPa}]$ & $c_{n}^{\max }[\mathrm{MPa}]$ \\
\hline 0 & 0.1 & 0.001 & 2.5 & 1.0 \\
1 & 0.2 & 0.001 & 5.0 & 2.0 \\
2 & 0.15 & 0.001 & 3.75 & 1.5 \\
3 & 0.067 & 0.001 & 1.67 & 0.67 \\
4 & 0.05 & 0.001 & 1.25 & 0.5 \\
5 & 0.4 & 0.00025 & 2.5 & 1.0 \\
6 & 0.2 & 0.0005 & 2.5 & 1.0 \\
7 & 0.05 & 0.002 & 2.5 & 1.0 \\
8 & 0.025 & 0.004 & 2.5 & 1.0 \\
\hline
\end{tabular}

peak behaviour of the overall microscale response is mostly related to the softening and damage at grain contacts. Increasing the interface softening slopes induces a more pronounced overall strain softening. Furthermore, the calibrated set of properties in Table 2 is used for all the following modelling.

It is also to mention that, regarding the mineralogical composition of the clay rock and the local clay fraction distribution in Fig. 6 (b) (Cosenza et al. 2015a,b), the considered EA has a clay content $(60 \%)$ close to the maximal content. Thus, the previous calibration could also have been done for lower clay contents. However, in a range of 40 to $60 \%$ (Fig. 6 (b)), the clay content does not affect significantly the calibration of grain interface properties. The vari- ability of material response to the mineral phase contents is studied in a following section.

\subsection{Variability of material response}

The material response to biaxial compression is now modelled for a set of 32 numerical microstructures described in sections 4.1 and 4.2, with parameters from Tab. 2. The stress-strain response curves are detailed in Fig. 10 for confining pressures of $\sigma_{3}=2$ and $12 \mathrm{MPa}$ and compared to experimental data. The results highlight the dispersion of the microscale behaviour related to the variability of the microstructures. The latter is related to the variability of the mineral phase contents, the method of EA generation, and the grain morphology as described in section 4 . The calibrated interface properties allow to obtain an accurate reproduction of the material response, especially in the vertical direction at high confinement.

\subsection{Sensitivity analyses}

The influences of several microscale characteristics on the material response and on its variability are investigated with sensitivity analyses. They are performed with a focus on 

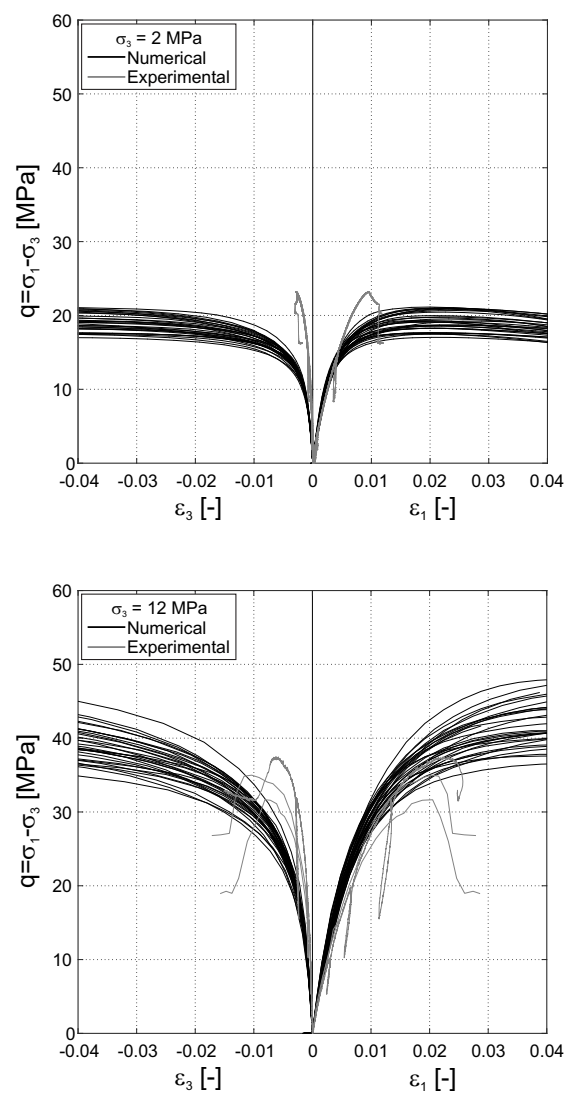

Fig. 10 Variability of deviatoric stress responses at microscale under biaxial compression related to microstructural characteristics (mineral contents, grain morphology, and inclusion positions). Comparison to experimental data at macroscale for $\sigma_{3}=2$ and $12 \mathrm{MPa}$.

the microstructure generation for identical micromechanical properties of Table 2 .

\subsubsection{Mineral phase contents}

The variability of the mineral area fractions of the generated set of microstructures (Fig. 6) is a source of variability of the microscale global behaviour. Amongst the minerals, pyrite is very stiff but is present in small quantities, quartz and carbonates have a similar stiffness, and the clay matrix has a low stiffness. Therefore, a low clay content, i.e. high content of mineral inclusions, leads to a stiffer response.

To enlighten this, EAs having different clay contents of $40 \%, 50 \%$, and $60 \%$ are considered. These values correspond to the minimal, mean, and maximal clay contents of the clay rock measured by Cosenza et al. (2015a) and reproduced in the EAs (Fig. 6). For each clay content, 10 EAs with identical mineral contents are biaxially loaded under a confinement of $\sigma_{3}=12 \mathrm{MPa}$. The numerical results are depicted in Fig. 11 with only the average response curve and the range for each set, i.e. the most resistant and least resistant responses. The mineral contents of quartz, carbonates, pyrite, and clay are respectively of 22-36-2-40\% in Fig. 11 (a), 18-30-2-50\% in Fig. 11 (b), which are the average values in Table 1, and 14-24-2-60\% in Fig. 11 (c).

From $60 \%$ to $40 \%$ of clay matrix, numerical results indicate that the average initial stiffness of the EAs (calculated between $\varepsilon_{1}=0 \%$ and $0.3 \%$ ) evolves from 3.5 to 5.5 $\mathrm{GPa}$. However, the mineral contents do not clearly affect the global peak strength which is mainly related to the grain interface properties.

\subsubsection{Microstructure generation}

Another origin of the microscale behaviour scattering is the randomness of EA generation by Voronoi tessellation. For identical mineral phase contents, the EAs have similar microstructural characteristics but with random allocations of the grain positions (sites of Voronoi cells) and variable grain morphology (size, elongation, orientation). To analyse the influence of the latter, 10 EAs with identical mineral contents are considered. The average values in Table 1 are firstly considered. The contents are $18 \%$ of quartz, $30 \%$ of carbonates, $2 \%$ of pyrite, and $50 \%$ of clay.

For these values and the micromechanical properties from Tab. 2, the numerical results of biaxial compressions under a confinement of $\sigma_{3}=12 \mathrm{MPa}$ are detailed in Fig. 11 (b) with only the average response curve and the range (most and least resistant responses). By comparison to the response dispersion in Fig. 10, which includes variable mineral contents, it can be seen that the variability of the microscale response for fixed mineral contents is limited but not zero. Similar observations can be done for other cases of constant mineral contents by comparing Fig. 11 (a) and (c) to Fig. 10.

It implies that the randomness of inclusion positions and grain morphology contributes to the microscale behaviour variability. This suggests that, if from a morphological point of view, the chosen size of the EAs is considered sufficient, this is not quite the case from the point of view of the homogenised mechanical response.

\subsubsection{Grain angularity}

The grain angularity also influences the microscale behaviour. It can be controlled in the EA generation by an optimisation of the roundness of the grains. The optimisation consists in a shape correction of the Voronoi cells related to a form of minimisation of the specific grain boundary (quadratic sum of the grains' boundaries lengths) and modulated by a coefficient $\eta$ between 0 (original cell, maximum angularity) and 1 (full optimisation, maximum roundness) (van den Eijnden et al. 2017).

Examples of microstructures composed of 250 cells with angular and rounded grains are available in Fig. 12 (a). The influence of grain angularity is analysed on microstructures having identical mineral contents (from Table 1), identical 


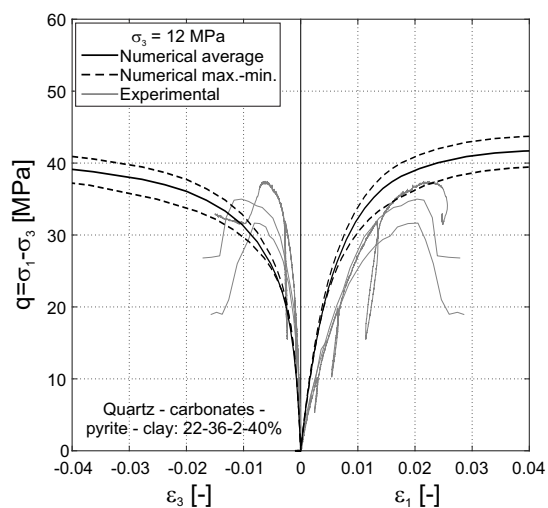

(a)

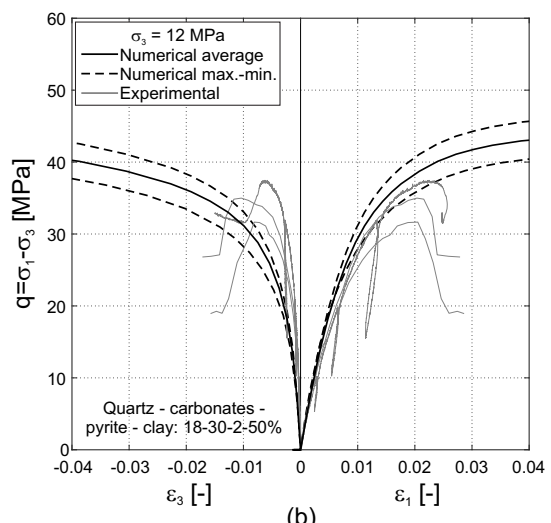

(b)

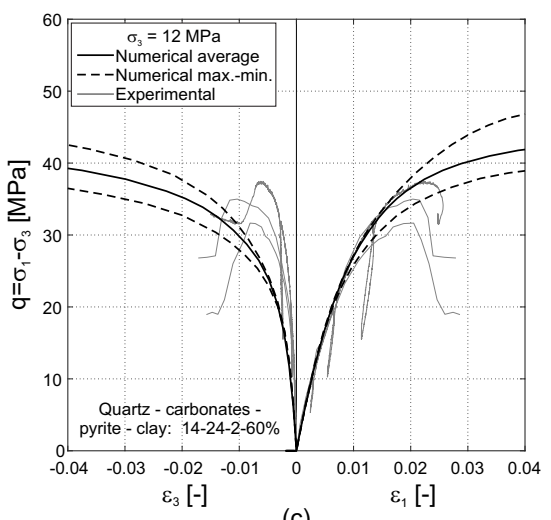

(c)

Fig. 11 Variability of microscale response, under biaxial compression, due to the randomness of EA generation by Voronoi tessellation and for several fixed mineral contents : (a) $40 \%$, (b) $50 \%$, and (c) $60 \%$ of clay.

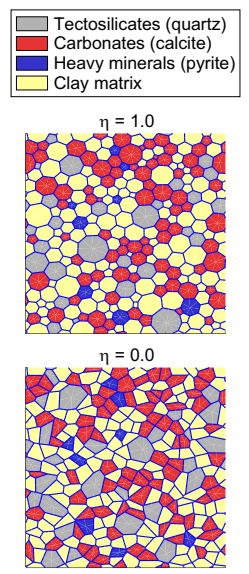

(a)

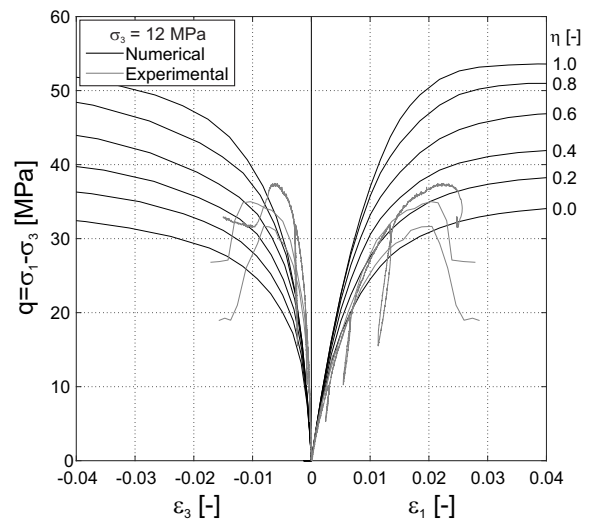

(b)
Fig. 12 Sensitivity of microscale response, under biaxial compression, to grain angularity: (a) microstructures and (b) material response for different angularities.

numbers and positions of inclusions (Fig. 12 (a)), as well as identical micromechanical properties (Table 2). The sensitivity of the overall microscale response to grain angularity under biaxial compression $\left(\sigma_{3}=12 \mathrm{MPa}\right)$ is illustrated in Fig. 12 (b). The results highlight that an increase of the grain roundness engenders an increase of both stiffness (slight increase) and peak strength. However, the roundness of grains reduces their elongations, which deteriorates the reproduction of the geometrical characteristics measured experimentally (Fig. 7 (a,c)) and the expected anisotropy. A deterioration is already observed for $\eta=0.4$. Consequently, a low value of $\eta=0.2$ is selected to reproduce correctly both grain morphology and material response.

\subsubsection{Characteristic size}

We have seen previously that the representativeness of the microstructure on morphological aspects is related to its size (section 4.2). This has led to the choice of EAs with 250 cells allowing the reproduction of the representative length of the microstructure $\left(L_{E A} \approx L_{R E V} \approx 100 \mu \mathrm{m}\right)$. Nevertheless, the effect of the microstructure size on the microscale mechanical response deserves to be studied.

It is analysed by considering EAs with an increasing number of Voronoi cells of 50, 100, and 250 cells (Fig. 13 (a)), giving $L_{E A}=50,70$, and $105 \mu \mathrm{m}$, respectively. All the other parameters are kept constant with mineral contents and micromechanical properties from Tables 1 and 2. The range of the response curves over 10 tested EAs for each case is illustrated in Fig. 13 (b) where the most and least resistant responses are represented for each set. One can observe that the microscale response is similar but the variability decreases with increasing size of the microstructures. It confirms that the partial lack of representativeness of the EA mechanical response is mitigated by the microstructure size. The dispersion of the responses for microstructures with 250 grains is relatively limited. It is considered that this number of 250 grains is a good compromise between representativeness, cost in terms of computation time in a double scale framework, and a certain dispersion. The latter reflects multiple forms of variability in the microstructure inherent in a natural material that encompasses a stochastic character.

\subsection{Small-scale deformation}

The microscale behaviour has been analysed in term of global stress-strain response of elementary areas. However, deformations at microscale occur within the EAs due to solicitations. To enlighten them, an analysis of the small-scale deformation is presented during a biaxial loading $\left(\sigma_{3}=12\right.$ $\mathrm{MPa})$.

\subsubsection{Grain movements and micro-damage}

Deformations are studied on one microstructure to enlighten grain movements and micro-damage of interfaces. The tested 


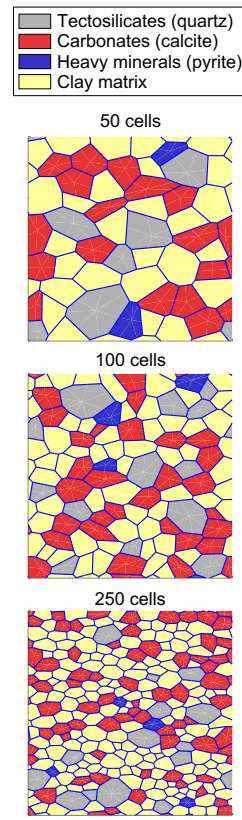

(a)

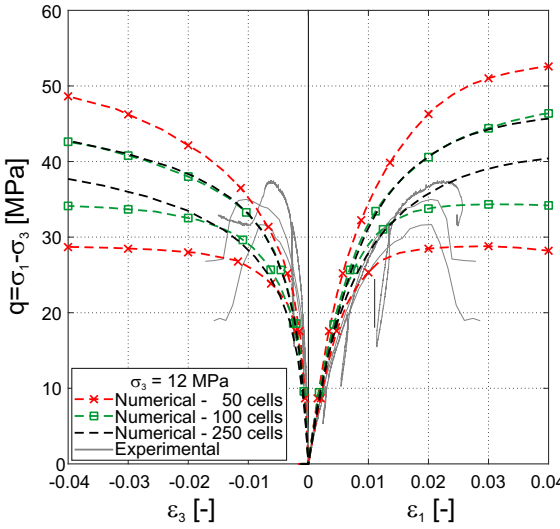

(b)
Fig. 13 Variability of microscale response, under biaxial compression, to microstructure size: (a) example of microstructures with three different numbers of grains and (b) material most and least resistant responses for the corresponding sets.

microstructure is the one on the bottom of Fig. 6 (a) with $60 \%$ clay content. The response curve is detailed in Fig. 14 (a) up to $\varepsilon_{1}=10 \%$ of vertical deformation. One can observe that the maximal shear strength is of $q^{\max }=40.6 \mathrm{MPa}$ and occurs for a vertical strain of $\varepsilon_{1}=3.4 \%$. Then, strain softening occurs with a reduction of the deviatoric stress in postpeak regime. This behaviour depends on the calibrated interface properties (Table 2).

The small-scale deformations are illustrated in Fig. 14 (b) for $\varepsilon_{1}=1 \%, 3 \%, 4 \%, 5 \%, 7 \%$, and $10 \%$ and indicated on the response curve of Fig. 14 (a). Some graphical symbols in Fig. 14 (b) indicate cohesion softening (partially damaged) and complete decohesion (fully damaged) of interfaces. Because the interface behaviour is defined, in the actual version, independently in tangential and normal directions, both components of damage can develop on the same interface. The representation of the grain interface state in Fig. 14 (b) includes both components in a global damage value. The latter is defined as the maximal value of the damage ratios of the two modes:

$\bar{D}=\max \left(D_{n}, D_{t}\right)$

where $D_{t / n}$ are the damage ratios in shear (tangential) and opening (normal) modes (Eq. 9 and 10). Thus, the global damage at grain contacts is represented in Fig. 14 (b) with a distinction between interfaces having reached cohesion softening $D_{t / n}^{0}<\bar{D}<1$ and complete decohesion $\bar{D}=1$ (fully damaged). Moreover, the size of the graphical symbols in Fig. 14 (b) are proportional to the global damage value $\bar{D}$.

One can observe that the deformation of the microstructure remains limited before peak stress $\left(\varepsilon_{1} \leq 3.4 \%\right)$. In prepeak regime, an interface softening, especially in shearing, initiates which induces a loss of linearity of the EA response. Then, an increase of damage and a coalescence of damaged interfaces occurs during the loading to form a micro fault that propagates through the whole EA. It induces a strain softening behaviour of the global response. In postpeak regime, interfaces attain complete decohesion in the fault. This generates important displacements between grains. Moreover, the micro fault pattern is constrained by periodic boundary conditions (Bilbie et al. 2008).

To better understand the grain interface behaviour, three damage indicators are studied during the vertical loading. The first in Fig. 15 (a) corresponds to the maximal damage ratios of all the interfaces in the EA, in both normal $D_{n}^{\max }$ and tangential $D_{t}^{\max }$ directions. It is therefore related to one interface which damage state is the highest in the whole EA. It is to recall that the damage ratios in the interfaces evolve from 0 at $\Delta u_{t / n}=0, D_{t / n}^{0}$ at the start of cohesion softening, to 1 at complete decohesion (Fig. 3, Eqs. 7 and 8). The second damage indicator in Fig. 15 (b) is the proportion of interfaces in the EA having reached softening and complete decohesion. It consists in a more general indicator of the overall damage state of the microstructure. The third indicator in Fig. 15 (c) corresponds to the proportion of interfaces which are more damaged in shear mode than in opening mode $D_{t}>D_{n}$, vice versa $D_{t}<D_{n}$, or which are still in elastic state with $D_{t}$ and $D_{n}<D_{t / n}^{0}$. It allows determining the dominant mode of deformation and damage at each grain contacts. In Fig. 15, the peak deviatoric stress of the EA mechanical response is also indicated to distinguish pre- and post-peak global microscale regimes.

The numerical results indicate that the interface behaviour remains globally elastic during the isotropic confining phase, even if a low interface softening develops (Fig. 15 (b-c)). At the end of the confining phase (beginning of the curves at $\left.\varepsilon_{1}=0\right)$, only a small proportion $(7.7 \%)$ of interfaces reaches softening $(6.1 \%$ in shear and $1.6 \%$ in opening, Fig. 15 (b)) with low damage ratios $\left(D_{t}^{\max }=3.21 E-3\right.$ and $D_{n}^{\max }=3.79 E-$ 3, Fig. 15 (a)).

During the vertical compression in pre-peak regime, the proportion of elastic interfaces decreases (Fig. 15 (c)); thus, the proportions of interfaces in softening mode increase (Fig. 15 (b)), together with an increase of the damage ratios (Fig. 15 (a)). This induces a loss of linearity of the EA response in pre-peak regime (Fig. 14 (a)). Besides, interfaces can be damaged in both directions simultaneously, as highlighted by the proportions in Fig. 15 (b). However, the results in Fig. 15 (c) indicate that most of the interfaces in the EA are more damaged in shear mode than in opening mode with 


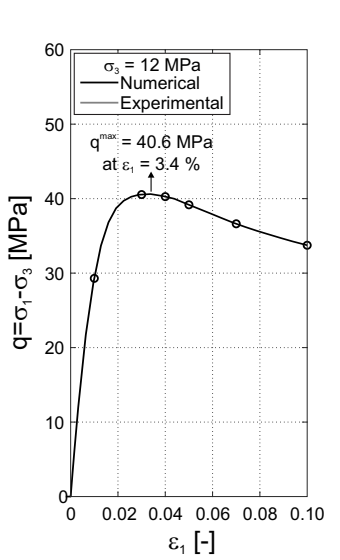

(a)
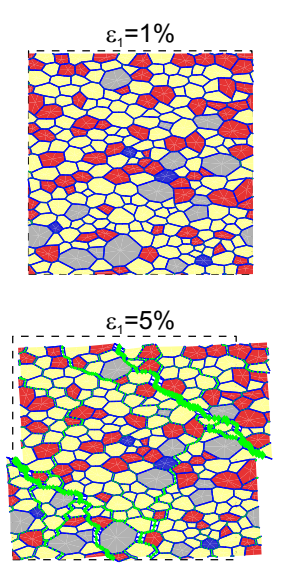

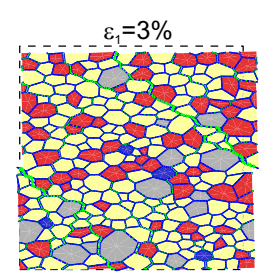

$\varepsilon_{1}=7 \%$

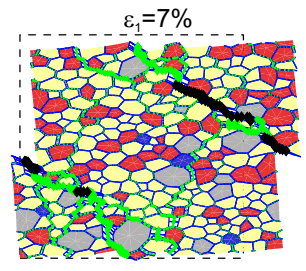

(b)

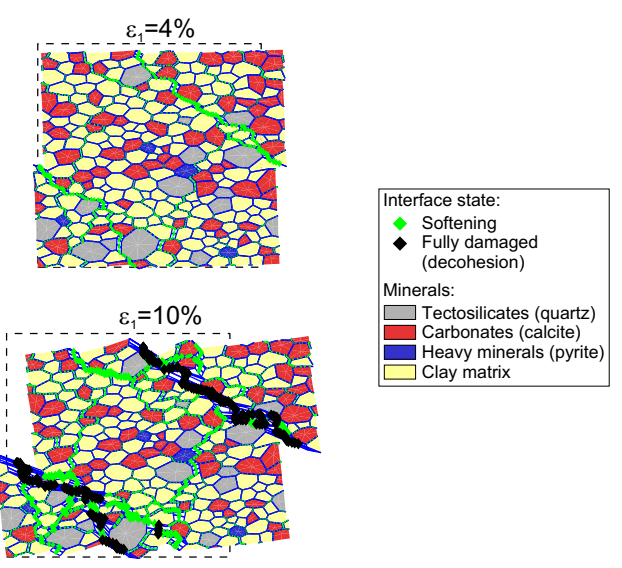

Fig. 14 Microstructural evolution by deformation process at grain scale: (a) material response and (b) appearance of microcracks under biaxial compression (true deformation).

$D_{t}>D_{n}$. In fact, all damage indicators are higher for the shearing (Fig. 15 (a-c)) which indicates that the softening in the tangential direction develops more rapidly than in the normal direction, under deviatoric loading of the EA. This is consistent with the observed interface cohesion softening and tangential relative displacements between grains in Fig. 14 (b).

Then, when the peak deviatoric stress is reached, only $3.4 \%$ of the interfaces remain elastic (Fig. 15 (c)). A proportion of $95.5 \%$ and $39.0 \%$ of interfaces are in softening mode in tangential and normal directions respectively (Fig. 15 (b)), with shearing the dominant mode of damage $\left(D_{t}>D_{n}\right.$ for $74.3 \%$ of interfaces, Fig. 15 (c)). However, the maximal damage ratios remain below a value of 1 (Fig. 15 (a)). Consequently, none of the interfaces in the EA attains a complete decohesion when the global strain softening regime starts. In post-peak regime, the first complete decohesion of interface in the tangential and normal directions $D_{t / n}^{\max }=1 \mathrm{oc}-$ curs respectively at $\varepsilon_{1}=6 \%$ and $7 \%$ of vertical deformation (Fig. 15 (a)). In terms of vertical deformations, this is far after the peak deviatoric stress of the EA response.

The proportions of interfaces reaching complete decohesion correspond to the difference between the two lines related to each interface direction in Fig. 15 (b). At the end of the loading at $\varepsilon_{1}=10 \%$, the proportions of interfaces in complete decohesion are of $3.9 \%$ in shear and $1.9 \%$ in opening modes. The fully damaged interfaces are visible in Fig. 14 (b). Therefore, even if a small proportion of interfaces reaches complete decohesion, they contribute significantly to the microscopic localised deformations in the EA. Moreover, the results confirm that shearing is the principal mode of deformation, softening, and damage at the grain contacts. It implies that the dominant relative movement between grains is sliding in the tangential direction to their contacts.
The results in Fig. 14 (b) also emphasise that the coalescence of the microcracks creates intergranular cracks with a certain roughness (which depends on the tessellation). This roughness makes that, for questions of geometric compatibility, some cracks only slide while others also open which generates a global dilatancy. This can be seen in Fig. 14 (b) where lateral deformation of the microstructure develops during the vertical loading. Thus, even if the mechanical behaviour of asperities is not directly modelled at grain contacts (in the interface constitutive behaviour), a dilative global material behaviour is reproduced.

\subsubsection{Variability of microcracking patterns}

The deformation pattern detailed in Fig. 14 (b) is related to one EA. However, various types of microstructures with identical micromechanical properties (Table 2) can exhibit various microcracking patterns. Several microstructures (of 250 cells and $50 \%$ of clay content) representing different degrees of angularity, elongation, and preferential orientation of grains, are presented in Fig. 16 (b) in their deformed states. These states are obtained after a biaxial compression under $\sigma_{3}=12 \mathrm{MPa}$ and up to $\varepsilon_{1}=10 \%$. At this point, all global mechanical responses have reached softening state related to the softening and damage at grain contacts. The response curves are illustrated in Fig. 16 (a).

The cases [1-2] in Fig. 16 are microstructures which have similar micromechanical characteristics (grain number, grain morphology, and microscale mechanical parameters) than the one in Fig. 14. Their microcracking patterns can therefore be compared. However, mineral contents and inclusion positions differ. Both microstructures [1] and [2] have a clay content of 50\% while the microstructure in Fig. 14 has a clay content of $60 \%$. Their material response in prepeak stress regime can therefore be compared to the results of Fig. $11(b, c)$. In post-peak softening regime, comparing 


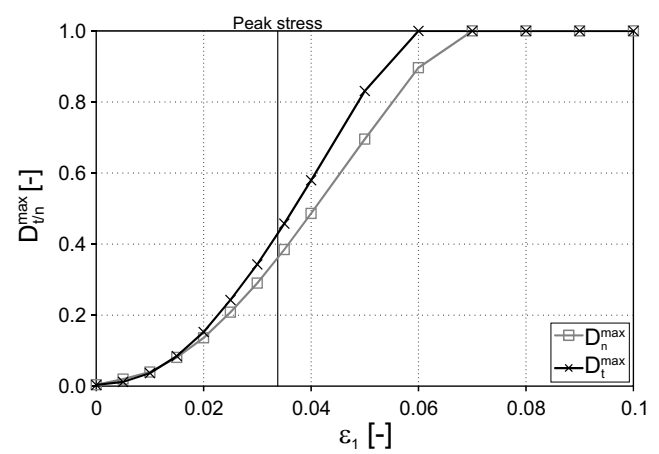

(a)

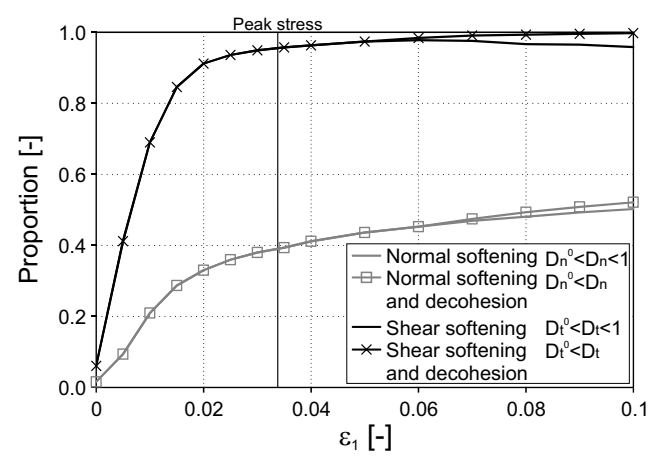

(b)

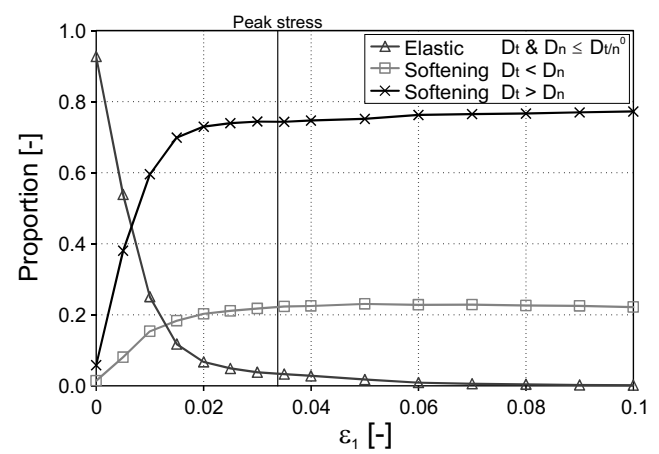

(c)

Fig. 15 Interface damage indicators of a microstructure under biaxial compression: (a) maximal damage ratios, (b) proportions of interfaces in softening and complete decohesion, and (c) proportions of interfaces more damaged in shear or in opening mode.

the results of Fig. 14 (b) and Fig. 16 (b) [1-2] indicates that the microcracking patterns are similar but with different inclinations and locations of the micro fault. The variability of the microcracking patterns is therefore related to the variability of inclusion positions. The latter differ due to the randomness of the generation method (random Voronoi tessellation). Furthermore, among these microstructures, the [2] exhibits a more distributed and lower damage of the interfaces and therefore a lower softening of the global microscale response (Fig. 16 (a) [1-2]).

The shape of the grains (angular, rounded, or elongated) also modifies the damage of the microstructure. The influ- ence on the rupture paths can be analysed. The case [3] in Fig. 16 considers rounded grains with the microstructure of Fig. 12 (a) for $\eta=1$. As detailed before, the peak strength of the global microscale response is increased for rounded grains (Fig. 16 (a) [3], Fig. 12). Moreover, the interface softening and damage are reduced by grain roundness which restricts the development of micro fault. The global softening of the EA is consequently also reduced. The case [4] considers an anisotropic microstructure composed of elongated grains oriented in the direction of the bedding planes (horizontal). It is obtained by defining a anisotropy stretch and a bedding orientation of the microstructure during its generation (van den Eijnden et al. 2017). In this particular case (compression perpendicular to the bedding), the elongation does not affect significantly the peak strength of the global microscale response, neither the orientation of microcracks (Fig. 16 [4]). However, in opposition to the previous case with rounded grains, it significantly increases the softening behaviour. Moreover, one notes a higher shearing of the microstructure than for other microstructures, indicating a significant rotation of the strain tensor principal directions.

Furthermore, the results in Fig. 14 to 16 highlight that microscale strain softening behaviour is related to grain movements, micro-damage, as well as initiation and propagation of intergranular micro fault (Galvanetto and Aliabadi 2009). Additionally, the shape of the grains influences the smallscale deformations, global peak strength, and strain softening behaviour.

\section{Conclusions}

The realism and representativeness of the microstructural behaviour modelling of a clay rock is improved by taking into account the microscale characteristics, including their heterogeneity and variability. The definition of numerical microstructures includes the properties of the mineralogical constituents, of the grain morphology, of the intergranular contacts and rupture modes, as well as the material characteristic size. Moreover, the influence of properties variability on the representativeness of the microscale behaviour is emphasised. By comparison to experimental data, the representativeness of generated microstructures is validated both geometrically and regarding the mechanical behaviour.

The modelling of the micromechanical behaviour focuses on the reproduction of global stress response and small-scale deformations. It emphasises the behaviour variability in relation to the microstructural properties. In terms of deviatoric stress response under biaxial compression, the variability of the overall behaviour is related to the random positions of inclusions and to the mineral contents. This variability decreases with increasing size of the microstructure. Besides, grain angularity and cohesive resistance at grain contacts influence the global material peak strength and softening. 


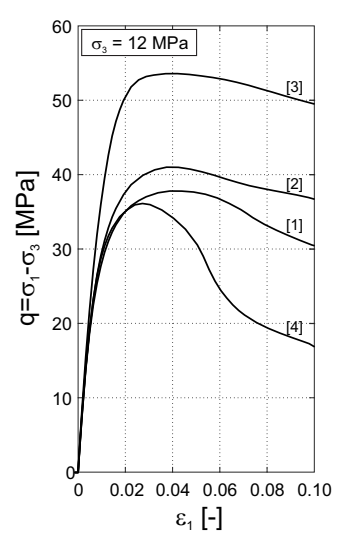

(a)
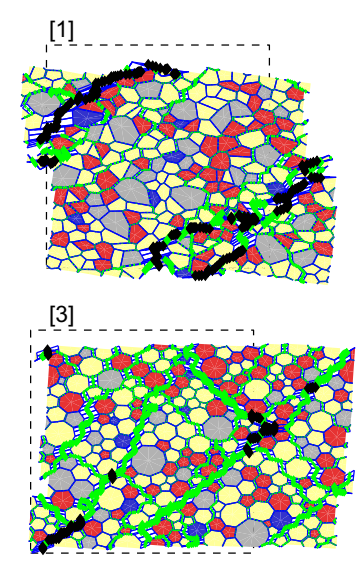

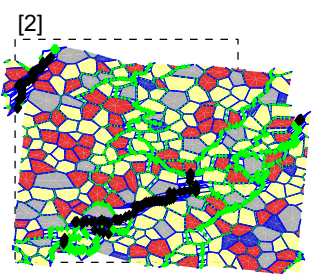

$[4]$

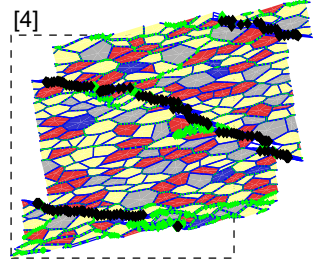

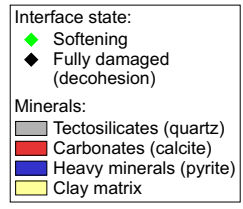

(b)

Fig. 16 Variability of deformations and damage for various types of microstructures under biaxial compression: (a) material response and (b) microckraking patterns for $\varepsilon_{1}=10 \%$.

Under deviatoric loading, numerical analyses of smallscale deformations demonstrate that shearing is the dominant mode of deformation at the grain contacts. In fact, during the loading, the proportions of contacts reaching softening or complete decohesion states are larger in shear mode than in opening mode. Thus, the dominant relative grain movements occur mainly by sliding along their interfaces. This deformation process induces a decohesion mechanism at some grain contacts which, in turn, engenders micro faulting. The decohesion is not generalised for all granular contacts but appears clearly in a localised manner for a small proportion of them. The micro faults develop between inclusions and clay matrix or within the clay matrix, as observed experimentally. Their appearance contributes significantly to localised deformations at small scale. Furthermore, the overall non-linearity and strain softening of the microstructure behaviour are due firstly to the initiation and development of micro-damage, then to the coalescence and propagation of intergranular micro faults. The variability of the microcracking patterns related to the microstructure variability (inclusion positions, morphology, etc.) is also studied. It emphasises the effect of the geometry of the potential cracking paths on the material behaviour. Finally, the enhanced homogenised small-scale response can serve as a more realistic constitutive law at larger-scale in the context of double-scale computing.

Acknowledgements The authors acknowledge the French programme NEEDS for its financial support to the project. The laboratoire 3SR is part of the LabEx Tec 21 (Investissements d'Avenir - grant agreement nANR-11-LABX-0030).

\section{Conflict of interest}

The authors declare that they have no conflict of interest.

\section{References}

Ahrens T (1995) Mineral physics and crystallography: a handbook of physical constants. 354p.

Andra (2005) Dossier 2005 Argile. Synthesis: Evaluation of the feasibility of a geological repository in an argillaceous formation, Meuse/Haute Marne site. Tech. rep., Paris, France

Armand G, Leveau F, Nussbaum C, de La Vaissiere R, Noiret A, Jaeggi D, Landrein P, Righini C (2014) Geometry and properties of the excavation-induced fractures at the Meuse/Haute-Marne URL drifts. Rock Mech Rock Eng 47(1):21-41

Armand G, Conil N, Talandier J, Seyedi DM (2017) Fundamental aspects of the hydromechanical behaviour of Callovo-Oxfordian claystone: From experimental studies to model calibration and validation. Comput Geotech 85:277-286

Bésuelle P, Chambon R, Collin F (2007) Switching mode of deformation in post-localization solutions with a quasi brittle material. $\mathrm{J}$ Mech Mater Struct 1(7):1115-1134

Bésuelle P, Andò E, Stamati O, Boller E (2019) Mesure de champs de déformation dans l'argillite du Callovo-Oxfordien à l'échelle du micron. In: $3^{\text {eme }}$ journées thématiques des Techniques d'imagerie pour la caractérisation des matériaux et des structures du génie civil, pp 1-2

Bilbie G, Dascalu C, Chambon R, Caillerie D (2008) Micro-fracture instabilities in granular solids. Acta Geotech 3(1):25-35

Cooper DW (1988) Random-sequential-packing simulations in three dimensions for spheres. Phys Rev A 38:522-524

Cosenza P, Prêt D, Giraud A, Hedan S (2015a) Effect of the local clay distribution on the effective elastic properties of shales. Mech Mater 84:55-74

Cosenza P, Prêt D, Zamora M (2015b) Effect of the local clay distribution on the effective electrical conductivity of clay rocks. J Geophys Res Solid Earth 120:145-168

Cosenza P, Fauchille AL, Prêt D, Hedan S, Giraud A (2019) Statistical representative elementary area of shale inferred by micromechanics. Int J Eng Sci 142:53-73

Croisé J, Schlickenrieder L, Marschall P, Boisson JY, Vogel P, Yamamoto S (2004) Hydrogeological investigations in a low permeability claystone formation: the Mont Terri Rock Laboratory. Phys Chem Earth 29(1):3-15

Desbois G, Höhne N, Urai JL, Bésuelle P, Viggiani G (2017) Deformation in cemented mudrock (Callovo-Oxfordian Clay) by microcracking, granular flow and phyllosilicate plasticity: insights from triaxial deformation, broad ion beam polishing and scanning elec- 
tron microscopy. Solid Earth 8(2):291-305

Desrues J, Argilaga A, Caillerie D, Combe G, Nguyen K, Richefeu V, Dal Pont S (2019) From discrete to continuum modelling of boundary value problems in geomechanics: an integrated FEMDEM approach. Int J Numer Anal Meth Geomech 43(5):919-955

van den Eijnden A, Bésuelle P, Chambon R, Collin F (2016) A FE ${ }^{2}$ modelling approach to hydromechanical coupling in crackinginduced localization problems. Int J Solids Struct 97-98:475-488

van den Eijnden A, Bésuelle P, Collin F, Chambon R, Desrues J (2017) Modeling the strain localization around an underground gallery with a hydro-mechanical double scale model; effect of anisotropy. Comput Geotech 85:384-400

Fauchille A, van den Eijnden A, Ma L, Chandler M, Taylor K, Madi K, Lee P, Rutter E (2018) Variability in spatial distribution of mineral phases in the Lower Bowland Shale, UK, from the mm- to $\mu \mathrm{m}$ scale: Quantitative characterization and modelling. Mar Petroleum Geol 92:109-127

Fauchille AL (2015) Déterminismes microstructuraux et minéralogiques de la fissuration hydrique dans les argilites de Tournemire : apports couplés de la pétrographie quantitative et de la corrélation d'images numériques. PhD thesis, Université de Poitiers, Poitiers

Feyel F, Chaboche JL (2000) $\mathrm{FE}^{2}$ multiscale approach for modelling the elastoviscoplastic behaviour of long fibre $\mathrm{SiC} / \mathrm{Ti}$ composite materials. Comput Methods Appl Mech Eng 183(3-4):309-330

French ME, Chester FM, Chester JS (2015) Micromechanisms of creep in clay-rich gouge from the central deforming zone of the san andreas fault. J Geophys Res Solid Earth 120(2):827-849

Frey J, Chambon R, Dascalu C (2013) A two-scale poromechanical model for cohesive rocks. Acta Geotech 8(2):107-124

Fritzen F, Böhlke T, Schnack E (2009) Periodic three-dimensional mesh generation for crystalline aggregates based on voronoi tessellations. Comput Mech 43(5):701-713

Galvanetto U, Aliabadi M (2009) Multiscale Modeling in Solid Mechanics: Computational Approaches. Imperial College Press, $334 \mathrm{p}$.

Gaucher E, Robelin C, Matray J, Négrel G, Gros Y, Heitz J, Vinsot A, Rebours H, Cassagnabère A, Bouchet A (2004) ANDRA underground research laboratory: Interpretation of the mineralogical and geochemical data acquired in the Callovian-Oxfordian formation by investigative drilling. Phys Chem Earth 29:55-77

Geers M, Yvonnet J (2016) Multiscale modeling of microstructure-property relations. MRS Bulletin 41(8):610-616

Ghosh S (2011) Micromechanical Analysis and Multi-Scale Modeling: Using the Voronoi Cell Finite Element Method. 730p.

Ghossein E, Lévesque M (2013) Random generation of periodic hard ellipsoids based on molecular dynamics: A computationallyefficient algorithm. J Comput Phys 253:471 - 490

Haines SH, Kaproth B, Marone C, Saffer D, van der Pluijm B (2013) Shear zones in clay-rich fault gouge: A laboratory study of fabric development and evolution. J Struct Geol 51:206 - 225

Hill R (1965) A self-consistent mechanics of composite materials. J Mech Phys Solids 13(4):213-222

Jorand R (2006) Etude expérimentale de la conductivité thermique: application au forage est205 du site de meuse/haute marne (andra). $\mathrm{PhD}$ thesis, University of Denis Diderot, Paris

Kickmaier W, McKinley I (1997) A review of research carried out in European rock laboratories. Nucl Eng Des 176(1-2):75-81

Klinkenberg M, Kaufhold S, Dohrmann R, Siegesmund S (2009) Influence of carbonate microfabrics on the failure strength of claystones. Eng Geol 107(1):42 - 54

Kouznetsova V, Brekelmans W, Baaijens F (2001) An approach to micro-macro modeling of heterogeneous materials. Comput Mech 27(1):37-48

Lubachevsky BD, Stillinger FH (1990) Geometric properties of random disk packings. J Stat Phys 60(5):561-583
Mandel J (1972) Plasticité classique et viscoplasticité, vol CISM Lecture Notes. Springer-Verlag, 187p.

Marinelli F, van den Eijnden A, Sieffert Y, Chambon R, Collin F (2016) Modeling of granular solids with computational homogenization: Comparison with Biot's theory. Finite Elem Anal Des 119:45-62

Massart TJ, Selvadurai APS (2012) Stress-induced permeability evolution in a quasi-brittle geomaterial. J Geophys Res Solid Earth 117(B7)

Morgenstern NR, Tchalenko JS (1967) Microscopic structures in kaolin subjected to direct shear. Géotechnique 17(4):309-328

M'Jahad S, Davy CA, Skoczylas F, Talandier J (2017) Characterization of transport and water retention properties of damaged CallovoOxfordian claystone. In: Radioactive Waste Confinement: Clays in Natural and Engineered Barriers, Geological Society of London

Nguyen T, Yvonnet J, Bornert M, Chateau C, Bilteryst F, Steib E (2017) Large-scale simulations of quasi-brittle microcracking in realistic highly heterogeneous microstructures obtained from micro ct imaging. Extr Mech Lett 17:50 - 55

Ortega J, Ulm FJ, Abousleiman Y (2007) The effect of the nanogranular nature of shale on their poroelastic behavior. Acta Geotech 2(3):155-182

Pardoen B, Collin F (2017) Modelling the influence of strain localisation and viscosity on the behaviour of underground drifts drilled in claystone. Comput Geotech 85:351-367

Pardoen B, Dal Pont S, Desrues J, Bésuelle P, Prêt D, Cosenza P (2018) Heterogeneity and variability of clay rock microstructure in a hydro-mechanical double scale FEM x FEM analysis. In: Giovine P, Mariano P, Mortara G (eds) Micro to MACRO Mathematical Modelling in Soil Mechanics. Trends in Mathematics, Birkhäuser, Cham, Springer, pp 247-256

Robinet JC (2008) Minéralogie, porosité et diffusion des solutés dans l'argilite du Callovo-Oxfordien de Bure (Meuse/Haute-Marne, France) de l'échelle centimétrique á micrométrique. $\mathrm{PhD}$ thesis, Université de Poitiers, Poitiers

Robinet JC, Sardini P, Coelho D, Parneix JC, Prêt D, Sammartino S, Boller E, Altmann S (2012) Effects of mineral distribution at mesoscopic scale on solute diffusion in a clay-rich rock: Example of the Callovo-Oxfordian mudstone (Bure, France). Water Resour Res 48(5)

Rutter EH, Maddock RH, Hall SH, White SH (1986) Comparative microstructures of natural and experimentally produced clay-bearing fault gouges. Pure Appl Geophys 124(1):3-30

Sammartino S, Bouchet A, Prêt D, Parneix JC, Tevissen E (2003) Spatial distribution of porosity and minerals in clay rocks from the Callovo-Oxfordian formation (Meuse/Haute-Marne, Eastern France)-implications on ionic species diffusion and rock sorption capability. Appl Clay Sci 23(1-4):157-166

Schröder J (2014) Plasticity and Beyond, vol 550, Springer, chap A numerical two-scale homogenization scheme: the $\mathrm{FE}^{2}$-method, pp $1-64$

Shen WQ, Shao JF (2014) A micro-macro model for porous geomaterials with inclusion debonding. Int J Dam Mech 24(7):1026-1046

Shen WQ, Shao JF (2015) A micromechanical model of inherently anisotropic rocks. Comput Geotech 65:73-79

Shen WQ, Shao JF, Kondo D, Gatmiri B (2012) A micro-macro model for clayey rocks with a plastic compressible porous matrix. Int J Plast 36:64-85

Smit R, Brekelmans W, Meijer H (1998) Prediction of the mechanical behavior of nonlinear heterogeneous systems by multi-level finite element modeling. Comput Methods Appl Mech Eng 155(1):181 $-192$

Sonon B, François B, Massart TJ (2015) An advanced approach for the generation of complex cellular material representative volume elements using distance fields and level sets. Comput Mech 56(2):221-242 
Stamati O, Roubin E, Andò E, Malecot Y (2019) Tensile failure of micro-concrete: from mechanical tests to fe meso-model with the help of x-ray tomography. Meccanica 54(4-5):707-722

Wang L, Bornert M, Héripré E, Chanchole S, Pouya A, Halphen B (2015) Microscale insight into the influence of humidity on the mechanical behavior of mudstones. J Geophys Res Solid Earth 120(5):3173-3186

Widom B (1966) Random sequential addition of hard spheres to a volume 44(10):3888-3894

Yamaji A, Masuda F (2005) Improvements in graphical representation of fabric data, showing the influence of aspect ratios of grains on their orientations. J Sediment Res 75(3):514-519

Yven B, Sammartino S, Geraud Y, Homand F, Villieras F (2007) Mineralogy, texture and porosity of Callovo-Oxfordian argillites of the Meuse/Haute-Marne region (eastern Paris Basin). Mém Soc géol France 178:73-90

Zaoui A (2002) Continuum micromechanics: Survey. J Eng Mech 128(8):808-816 